This item was submitted to Loughborough's Research Repository by the author.

Items in Figshare are protected by copyright, with all rights reserved, unless otherwise indicated.

\title{
On uniqueness and trapped modes in the water-wave problem for vertical
} barriers

PLEASE CITE THE PUBLISHED VERSION

LICENCE

CC BY-NC-ND 4.0

\section{REPOSITORY RECORD}

Kuznetsov, N., P. Mclver, and C.M. Linton. 2019. "On Uniqueness and Trapped Modes in the Water-wave Problem for Vertical Barriers”. figshare. https://hdl.handle.net/2134/836. 


\title{
On uniqueness and trapped modes in the water-wave problem for vertical barriers
}

\author{
N. Kuznetsov ${ }^{1}$, P. McIver ${ }^{2}$ and C. M. Linton ${ }^{2}$ \\ ${ }^{1}$ Laboratory for Mathematical Modelling of Wave Phenomena, \\ Institute of Problems in Mechanical Engineering, Russian Academy of Sciences, \\ V.O., Bol'shoy pr. 61, St Petersburg 199178, RF \\ ${ }^{2}$ Department of Mathematical Sciences, Loughborough University, \\ Loughborough, Leics, LE11 3TU, UK
}

\begin{abstract}
Uniqueness in the linearised water-wave problem is considered for a fluid layer of constant depth containing two, three or four vertical barriers. The barriers are parallel, of infinite length in a horizontal direction, and may be surface-piercing and/or bottom mounted and may have gaps. The case of oblique wave incidence is included in the theory.

A solution for a particular geometry is unique if there are no trapped modes, that is no free oscillations of finite energy. Thus, uniqueness is established by showing that an appropriate homogeneous problem has only the trivial solution. Under the assumption that at least one barrier does not occupy the entire fluid depth, the following results have been proven: for any configuration of two barriers the homogeneous problem has only the trivial solution for any frequency within the continuous spectrum; for an arbitrary configuration of three barriers the homogeneous problem has only the trivial solution for certain ranges of frequency within the continuous spectrum; for threebarrier configurations symmetric about a vertical line, it is shown that there are no correspondingly symmetric trapped modes for any frequency within the continuous spectrum; for four-barrier configurations symmetric about a vertical line, the homogeneous problem has only the trivial solution for certain ranges of frequency within the continuous spectrum.

The symmetric four-barrier problem is investigated numerically and strong evidence is presented for the existence of trapped modes in both finite and infinite depth. The trapped mode frequencies are found for particular geometries that are in agreement with the uniqueness results listed above.
\end{abstract}




\section{Introduction}

Over many years the problem of uniqueness of the solution to the linearised water-wave problem for time-harmonic motions has received much attention. Considerable effort has been directed towards attempts at obtaining a general uniqueness theorem, but without success. In the meantime, many partial results have been obtained with restrictions to particular classes of geometry or ranges of frequency. The reason for the lack of a general theorem became clear when McIver (1996) provided a counterexample which demonstrated that the problem need not be unique. For certain pairs of surface-piercing structures and for certain discrete frequencies, she was able to construct a solution of the homogeneous water-wave problem that has finite energy; such solutions are generally termed 'trapped modes'. The examples of trapped modes constructed by McIver (1996) and extended by Kuznetsov \& Porter (1999) have undermined the previously dominant view that there is uniqueness in the water-wave problem for all frequencies and for all geometries. There are important consequences for geometries with the non-uniqueness property. In particular, the non-homogeneous water-wave problem can have no solution for some types of forcing; for example, McIver (1997) has shown that the heave potential may not exist and in other situations there is more than one solution at the frequency at which non-trivial solutions of the homogeneous problem occur.

Since there are examples of non-uniqueness for geometries separating a portion of the free surface from infinity, it is natural to study in detail what are perhaps the simplest geometries of this type, namely configurations of two or more surface-piercing vertical barriers. This is the main topic of the work reported here where uniqueness results are presented for two, three and four barriers and numerical evidence is presented for the existence of trapped modes in the presence of four vertical barriers.

Levine \& Rodemich (1958) gave an explicit solution for the two-dimensional problem of scattering by a pair of identical vertical barriers that is valid for all frequencies. This is the only known explicit solution for two surface-piercing structures. Unfortunately, the solution is complicated and difficult to interpret. Subsequently, Evans \& Morris (1972) formulated the problem in terms of integral equations and, quite remarkably, showed that it is possible for such a pair of barriers to completely reflect an incident wave at certain discrete frequencies. In section 3 of the current work it is proven that the solution of the two-dimensional problem for any pair of surface-piercing barriers is unique at all frequencies. Thus, the uniqueness of Levine \& Rodemich's solution and 
the unique solvability of the integral equations obtained for the same problem by Evans \& Morris (1972) are established.

An explicit solution for the related two-dimensional problem of scattering by two vertical barriers extending downwards indefinitely from points the same distance beneath the free surface was obtained by Jarvis (1971). The solution is similar to, but simpler than, the solution of Levine \& Rodemich (1958) for two surface-piercing barriers. The uniqueness theorem of section 3 covers this case as well.

The ideas used in the uniqueness proof for two barriers are readily extended to three barriers and symmetric configurations of four barriers and this is done in sections 4 and 5. However, the proofs now apply to only certain configurations or ranges of frequency thus leaving open the possibility that trapped modes may exist for these geometries.

The possibility that a structure may totally reflect waves has led to speculation that this may be used as a basis for the construction of trapped modes. The following heuristic argument is reproduced verbatim from Evans \& Porter (1998). 'Consider, in two dimensions, the scattering of a plane wave by one or more fixed rigid bounded obstacles. The resulting reflection coefficient, $R$, depends on wave frequency and body geometry and satisfies $|R| \leq 1$. Suppose for a particular wave frequency and geometry, $|R|=1$, so that the plane wave is totally reflected. Placing an identical mirror-image configuration of bodies at a position sufficiently far downstream for local effects to be negligible, will result in the totally reflected plane wave in its turn being totally reflected and so on. By suitable choice of spacing of the two configurations it is possible to construct a standing wave oscillation in the region between the configurations and only a rapidly decaying local field outside.'

A number of geometries are known to exist for which $|R|=1$ (or alternatively $T=0$, where $T$ is the transmission coefficient). For instance, Parsons \& Martin (1994) showed numerically that a surface-piercing flat plate inclined to the vertical has this property. Subsequently, Linton \& Kuznetsov (1997) presented strong numerical evidence that waves can be trapped by a pair of such plates, and the frequencies of the modes are closely correlated with the frequencies at which $T=0$ for a single plate. The work of Linton \& Kuznetsov (1997) suggests that vertical barriers may be the only type of a symmetric, surface-piercing, barrier pair with the uniqueness of solution property.

As mentioned above, another configuration for which $T=0$ is a pair of suitably spaced, thin, vertical, surface-piercing barriers. This suggests that a geometry consisting of two pairs of such barriers may support trapped modes. In the present work, accurate computations using the method of Porter \& Evans (1995) for finite depth and that of Linton \& Kuznetsov (1997) for infinite depth 
are used to demonstrate very convincingly that trapped modes exist in the presence of two pairs of vertical barriers. The trapped-mode frequencies are close to the frequencies at which $T=0$ for a single pair of barriers and lie in the frequency bands for which the theorem on four barriers does not establish uniqueness.

The plan of the paper is as follows. The problem is stated and the governing equations presented in section 2. In sections 3,4 , and 5 uniqueness theorems are presented respectively for two, three and four vertical barriers. Numerical evidence for the existence of trapped modes in the presence of four barriers is presented in section 6. Formulations valid for finite and infinite depth are described in sections 6.1-6.2 and the results are given in section 6.3.

\section{Statement of the problem}

An inviscid, incompressible fluid occupies a layer of mean depth $h$ (possibly infinite) bounded below by a rigid bed and above by a free surface. Cartesian coordinates $x, y$ and $z$ are chosen with $y$ measured vertically upwards from the mean free surface. This paper is concerned with solutions of the linearised water-wave problem that have a velocity potential in the form $\phi(x, y) \mathrm{e}^{\mathrm{i}(\ell z-\omega t)}$; such solutions are time-harmonic with angular frequency $\omega$ and periodic in the $z$ direction with corresponding wavenumber $\ell$ (possibly zero).

Let $L$ denote the strip $\{-\infty<x<\infty ;-h<y<0\}, F$ the free surface, $B$ the bed $\{-\infty<$ $x<\infty ; y=-h\}$, and $S$ the boundary curve in the $(x, y)$ plane of any structures within the fluid; the fluid domain $W$ is therefore that part of $L$ outside $\bar{S}$. The potential $\phi$ satisfies

$$
\left(\nabla^{2}-\ell^{2}\right) \phi=0 \quad \text { in } \quad W
$$

and the boundary condition

$$
\phi_{y}-K \phi=0 \quad \text { on } \quad F
$$

where $K=\omega^{2} / g$ and $g$ is the acceleration due to gravity. There can be no flow through the rigid bed so that

$$
\phi_{y}=0 \quad \text { on } B
$$

and there must be no flow through the surface of any structure within the fluid so that

$$
\phi_{n}=0 \text { on } S,
$$


where $n$ denotes a coordinate measured normal to $S$. The present work is concerned with problems involving vertical barriers so that $S$ will be a union of lines on constant values of $x$.

For fixed $\ell$, the problem (1)-(4) defines an eigenvalue problem where $K$ is the spectral parameter. For a solution $\phi$ corresponding to a particular $K$, the quantity

$$
H[\phi ; K]=\int_{W}|\nabla \phi|^{2} \mathrm{~d} x \mathrm{~d} y+K \int_{F}|\phi|^{2} \mathrm{~d} x
$$

is proportional to the time-averaged energy of the motion per unit length of the $z$ axis. If, for a non-zero $\phi, H[\phi ; K]$ is unbounded then $K$ belongs to the continuous spectrum of the relevant operator, while if $H[\phi ; K]<\infty$ then $K$ is a point eigenvalue and the corresponding eigenfunction $\phi$ describes a so-called 'trapped mode'.

The continuous spectrum corresponds to those values of $K$ for which propagating waves are possible at infinity. As $x \rightarrow \pm \infty$, radiated-wave solutions of equations (1)-(3) are of the form

$$
\phi=C_{ \pm} \mathrm{e}^{\mathrm{i} \alpha x} \cosh k(y+h)+O\left(\left[x^{2}+y^{2}\right]^{-1 / 2}\right)
$$

where $k$ is the unique positive root of

$$
K=k \tanh k h
$$

and $\alpha=\sqrt{k^{2}-\ell^{2}}$ is real. Thus, the continuous spectrum corresponds to $k>\ell$ which is equivalent to $K>\ell \tanh \ell h$. Note that $k \rightarrow K$ in the deep water limit $h \rightarrow \infty$ and in this case $\cosh k(y+h)$ must be replaced by $\mathrm{e}^{K y}$ in (6).

For the solutions of (1)-(4) to have finite energy, it is sufficient for the radiation condition,

$$
\phi_{x} \mp \mathrm{i} \alpha \phi=o(1) \quad \text { as } \quad x \rightarrow \pm \infty
$$

to hold. Another condition arises from the fact that the boundary of $W$ is not smooth because of the presence of the submerged barrier tips and any corners between the barriers and $F$ or $B$. Thus, there is the additional requirement that

$$
\phi \in H_{\mathrm{loc}}^{1}(W)
$$

that is for every open set $E \subset W$ such that $\bar{E}$ is a compact subset of $\bar{L}$,

$$
\int_{E}|\phi|^{2} \mathrm{~d} x \mathrm{~d} y+\int_{E}|\nabla \phi|^{2} \mathrm{~d} x \mathrm{~d} y<\infty
$$

Equations (1)-(4) and (8) together with condition (9) constitute the statement of the homogeneous water-wave problem for vertical barriers that is to be investigated here. 


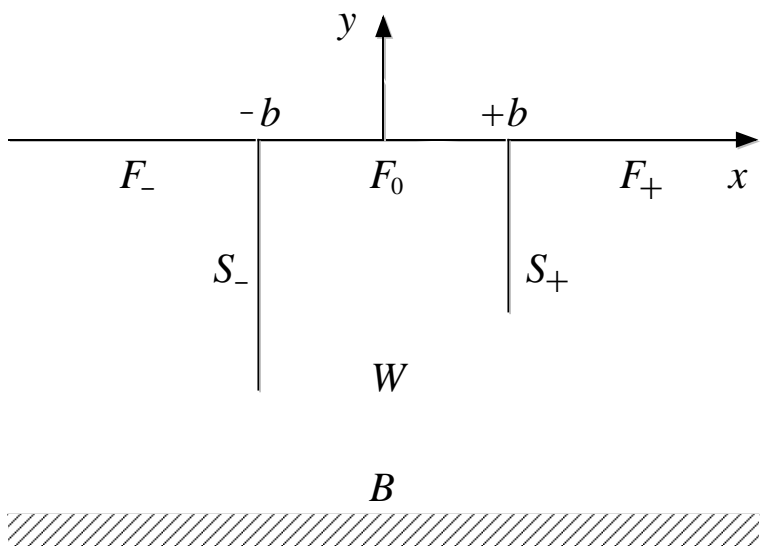

Figure 1: Definition sketch for two-barrier configuration.

We conclude this section with some simple consequences of (8) and (9). Using asymptotic expansions at a corner for solutions of elliptic boundary value problems (see Kozlov, Maz'ya \& Rossman (1997) for a general exposition or Wigley (1964) where particular formulae sufficient for the present work are given), one arrives at the following properties of the velocity potential satisfying (1)-(4) and (9). It is continuous at the submerged barrier tips and for each tip

$$
|\nabla \phi(x, y)|=O\left(\rho^{-1 / 2}\right) \quad \text { as } \quad \rho \rightarrow 0,
$$

where $\rho$ is the distance of a point in the fluid from that tip. Also, in each corner between $F$ or $B$ and a barrier, $\phi$ is continuous up to the boundary of the water domain and

$$
|\nabla \phi(x, y)|=O\left(r^{-1+\delta}\right) \quad \text { as } \quad r \rightarrow 0,
$$

where $r$ is the distance of a point in the fluid from that corner and $\delta>0$.

The second corollary is concerned with the behaviour of $\phi$ at infinity. It is well known (see for example Simon \& Ursell 1984) that $C_{+}=C_{-}=0$ in (6) for a solution to the homogeneous water-wave problem and hence $\phi \in H^{1}(W)$ so that the total energy of the fluid motion satisfies

$$
H[\phi ; K]<\infty .
$$

\section{A uniqueness theorem for two barriers}

Consider the two-barrier configuration sketched in figure 1. Two rigid vertical barriers are immersed in a fluid of depth $h$ so that their wetted surfaces occupy the lines $S_{ \pm}=\left\{x= \pm b ;-a_{ \pm}<y<0\right\}$, 
$a_{ \pm}>0$. The free surface of the fluid is $F=\{|x| \neq b ; y=0\}=F_{-} \cup F_{0} \cup F_{+}$, where the portions of the free surface that extend to infinity are $F_{ \pm}=\{ \pm x>b ; y=0\}$ and the portion between the barriers is $F_{0}=\{|x|<b ; y=0\}$. For the particular case of two barriers, the structural boundary condition (4) becomes

$$
\phi_{x}(+b \pm 0, y)=0 \quad \text { on } \quad S_{+} \quad \text { and } \quad \phi_{x}(-b \pm 0, y)=0 \quad \text { on } \quad S_{-} .
$$

The aim is to establish that the problem defined by equations (1)-(3), (8)-(9), and (14) has only the trivial solution for the continuous spectrum $K>\ell \tanh \ell h$. This is done by constructing an inequality which can be satisfied only if $\phi=0$. First of all it is convenient to establish some properties of

$$
w(x)=\int_{-h}^{0} \phi(x, y) \cosh k(y+h) \mathrm{d} y
$$

where $k$ is the unique positive root of (7). This function (referred to as the simple wave of order zero by John 1950) is given for all $x \in F$ since the barriers are assumed to be vertical. Differentiation with respect to $x$ and use of (1) gives

$$
w_{x x}(x)=\int_{-h}^{0}\left[\ell^{2} \phi(x, y)-\phi_{y y}(x, y)\right] \cosh k(y+h) \mathrm{d} y
$$

and so, by integration by parts and equations (2), (3) and (7),

$$
\begin{aligned}
w_{x x}-\ell^{2} w & =-\left[\phi_{y}(x, 0) \cosh k h-k \int_{-h}^{0} \phi_{y}(x, y) \sinh k(y+h) \mathrm{d} y\right] \\
& =-\left[K \phi(x, 0) \cosh k h-k \phi(x, 0) \sinh k h+k^{2} \int_{-h}^{0} \phi(x, y) \cosh k(y+h) \mathrm{d} y\right] \\
& =-k^{2} w(x)
\end{aligned}
$$

and hence

$$
w_{x x}+\alpha^{2} w=0 .
$$

The general solution of (18) is

$$
w(x)=C_{1} \cos \alpha x+C_{2} \sin \alpha x
$$

and hence from the radiation condition (8),

$$
w(x)=0 \quad \text { on } \quad F_{ \pm}
$$


(see John 1950, pp. 79-82 for details).

Now $\phi_{x}(x, y)$ is continuous across $x= \pm b$ for $y \neq-a_{ \pm}$. For $-a_{ \pm}<y<0$ this follows from (14) and for $-h<y<-a_{ \pm}$it is a consequence of analyticity of solutions to (1). This continuity of $\phi_{x}$ together with (11) and (12) imply that $w_{x}$ is continuous across $x= \pm b$. Hence, from (18) and (20),

$$
w_{x}=0 \quad \text { for } \quad|x|=b
$$

and thus on $F_{0}$

$$
w(x)=C_{1} \cos \alpha x \quad \text { with } \quad \alpha=m \pi / b, \quad m=1,2, \ldots,
$$

or

$$
w(x)=C_{2} \sin \alpha x \quad \text { with } \quad \alpha=(2 m-1) \pi / 2 b, \quad m=1,2, \ldots,
$$

so that only values of $K$ arising from $\alpha=n \pi / 2 b, n=1,2, \ldots$, can be eigenvalues of the problem (1)-(3), (8)-(9), and (14). From the above it follows immediately that

$$
\int_{F_{0}}\left|w_{x}\right|^{2} \mathrm{~d} x=\alpha^{2} \int_{F_{0}}|w|^{2} \mathrm{~d} x .
$$

The properties of $w$ are now used to establish certain bounds for $\phi$. Following John (1950), we note that for $(x, 0) \in F_{ \pm}$equation (20) gives

$$
\int_{-h}^{0} \phi(x, y) \cosh k(y+h) \mathrm{d} y=0
$$

and hence

$$
\phi(x, 0) \sinh k h=\int_{-h}^{0} \phi_{y}(x, y) \sinh k(y+h) \mathrm{d} y
$$

so that

$$
|\phi(x, 0) \sinh k h|^{2} \leq\left(\int_{-h}^{0}\left|\phi_{y}(x, y)\right|^{2} \mathrm{~d} y\right)\left(\int_{-h}^{0} \sinh ^{2} k(y+h) \mathrm{d} y\right)
$$

and, after evaluation of the last integral, this becomes

$$
K|\phi(x, 0)|^{2} \leq \frac{1}{2} \int_{-h}^{0}\left|\phi_{y}(x, y)\right|^{2} \mathrm{~d} y .
$$

Integration over the intervals $|x|>b$ gives

$$
K \int_{F_{+} \cup F_{-}}|\phi(x, 0)|^{2} \mathrm{~d} x \leq \frac{1}{2} \int_{W_{+} \cup W_{-}}\left|\phi_{y}(x, y)\right|^{2} \mathrm{~d} x \mathrm{~d} y
$$


where $W_{ \pm}=\{|x|>b ;-h<y<0\}$ are the fluid regions below $F_{ \pm}$.

Attention is now turned to obtaining a similar bound for the fluid region bounded above by $F_{0}$. Integration by parts in the definition (15) of $w$ gives

$$
\phi(x, 0) \sinh k h=k w(x)+\int_{-h}^{0} \phi_{y}(x, y) \sinh k(y+h) \mathrm{d} y \quad \text { for } \quad(x, 0) \in F_{0} .
$$

Now for $\lambda>0$

$$
(p+q)^{2}=p^{2}+q^{2}+2\left(\lambda^{1 / 2} p\right)\left(\lambda^{-1 / 2} q\right) \leq(1+\lambda) p^{2}+\left(1+\lambda^{-1}\right) q^{2},
$$

where the last inequality follows from the obvious relation $\left(\lambda^{1 / 2} p-\lambda^{-1 / 2} q\right)^{2} \geq 0$. Thus

$$
|\phi(x, 0) \sinh k h|^{2} \leq(1+\lambda) k^{2}|w(x)|^{2}+\left(1+\lambda^{-1}\right) \frac{\sinh ^{2} k h-K h}{2 K} \int_{-h}^{0}\left|\phi_{y}(x, y)\right|^{2} \mathrm{~d} y,
$$

where $\lambda$ is to be chosen later for convenience. From (15)

$$
|w(x)|^{2} \leq \frac{\sinh ^{2} k h+K h}{2 K} \int_{-h}^{0}|\phi(x, y)|^{2} \mathrm{~d} y
$$

and

$$
\left|w_{x}(x)\right|^{2} \leq \frac{\sinh ^{2} k h+K h}{2 K} \int_{-h}^{0}\left|\phi_{x}(x, y)\right|^{2} \mathrm{~d} y .
$$

Integration of (32) along $F_{0}$ gives

$$
K \int_{F_{0}}|\phi|^{2} \mathrm{~d} x \leq(1+\lambda) \frac{\ell^{2}+\alpha^{2}}{\sinh ^{2} k h} K \int_{F_{0}}|w|^{2} \mathrm{~d} x+\left(1+\lambda^{-1}\right) \frac{\sinh ^{2} k h-K h}{2 \sinh ^{2} k h} \int_{W_{0}}\left|\phi_{y}\right|^{2} \mathrm{~d} x \mathrm{~d} y
$$

and integration of (33) along $F_{0}$ gives

$$
\int_{F_{0}}|w|^{2} \mathrm{~d} x \leq \frac{\sinh ^{2} k h+K h}{2 K} \int_{W_{0}}|\phi|^{2} \mathrm{~d} x \mathrm{~d} y,
$$

where $W_{0}=\{|x|<b ;-h<y<0\}$ is the portion of the fluid domain lying directly beneath $F_{0}$. Combination of the last two inequalities with (24) and then with (34) gives

$$
\begin{aligned}
K \int_{F_{0}}|\phi|^{2} \mathrm{~d} x & \leq(1+\lambda) \frac{\sinh ^{2} k h+K h}{2 \sinh ^{2} k h} \ell^{2} \int_{W_{0}}|\phi|^{2} \mathrm{~d} x \mathrm{~d} y \\
& +(1+\lambda) \frac{K}{\sinh ^{2} k d} \int_{F_{0}}\left|w_{x}\right|^{2} \mathrm{~d} x+\left(1+\lambda^{-1}\right) \frac{\sinh ^{2} k h-K h}{2 \sinh ^{2} k h} \int_{W_{0}}\left|\phi_{y}\right|^{2} \mathrm{~d} x \mathrm{~d} y \\
& \leq(1+\lambda) \frac{\sinh ^{2} k h+K h}{2 \sinh ^{2} k h} \int_{W_{0}}\left(\ell^{2}|\phi|^{2}+\left|\phi_{x}\right|^{2}\right) \mathrm{d} x \mathrm{~d} y \\
& +\left(1+\lambda^{-1}\right) \frac{\sinh ^{2} k h-K h}{2 \sinh ^{2} k h} \int_{W_{0}}\left|\phi_{y}\right|^{2} \mathrm{~d} x \mathrm{~d} y .
\end{aligned}
$$


The choice

$$
\lambda=\frac{\sinh ^{2} k h-K h}{\sinh ^{2} k h+K h},
$$

which is positive in view of (7), simplifies the last inequality to

$$
K \int_{F_{0}}|\phi|^{2} \mathrm{~d} x \leq \int_{W_{0}}\left(\ell^{2}|\phi|^{2}+|\nabla \phi|^{2}\right) \mathrm{d} x \mathrm{~d} y .
$$

Finally, combination of (39) with the corresponding inequality (29) for the exterior domains yields

$$
K \int_{F}|\phi|^{2} \mathrm{~d} x \leq \frac{1}{2} \int_{W_{+} \cup W_{-}}\left|\phi_{y}\right|^{2} \mathrm{~d} x \mathrm{~d} y+\int_{W_{0}}\left(\ell^{2}|\phi|^{2}+|\nabla \phi|^{2}\right) \mathrm{d} x \mathrm{~d} y .
$$

Comparison of this with the energy identity

$$
K \int_{F}|\phi|^{2} \mathrm{~d} x=\int_{W}\left(\ell^{2}|\phi|^{2}+|\nabla \phi|^{2}\right) \mathrm{d} x \mathrm{~d} y
$$

which is a consequence of Green's formula, leads to the inequality

$$
\int_{W_{+} \cup W_{-}}\left(\frac{1}{2}|\nabla \phi|^{2}+\ell^{2}|\phi|^{2}\right) \mathrm{d} x \mathrm{~d} y \leq 0 .
$$

It immediately follows that $\phi \equiv 0$ in $W_{+} \cup W_{-}$and as $\phi$ is analytic it therefore vanishes throughout $W$.

Thus, the following uniqueness theorem has been proven.

For the geometry shown in figure 1 the two-barrier problem defined by equations (1)-(3), (8)-(9), and (14) has only the trivial solution for $K>\ell \tanh \ell h$.

The above result holds for more geometries than that in figure 1. The proof does not depend in any way on the positions of the barriers on the lines $x= \pm b$. Thus the water-wave problem under consideration has only the trivial solution for any configuration of barriers lying on the lines $x= \pm b$. The only requirement is that at least one of the lines has one or more gaps as the continuation argument following equation (42) breaks down without a gap in at least one of the lines.

\section{A uniqueness theorem for three barriers}

Consider now the three-barrier configuration sketched in figure 2. Three rigid vertical barriers are immersed in a fluid of depth $h$ so that their wetted surfaces occupy the lines $S_{ \pm}=$ $\left\{x=b_{ \pm} ;-a_{ \pm}<y<0\right\}$ and $S_{0}=\left\{x=0 ;-a_{0}<y<0\right\}$, with $a_{ \pm}, a_{0}>0$. The free surface of 


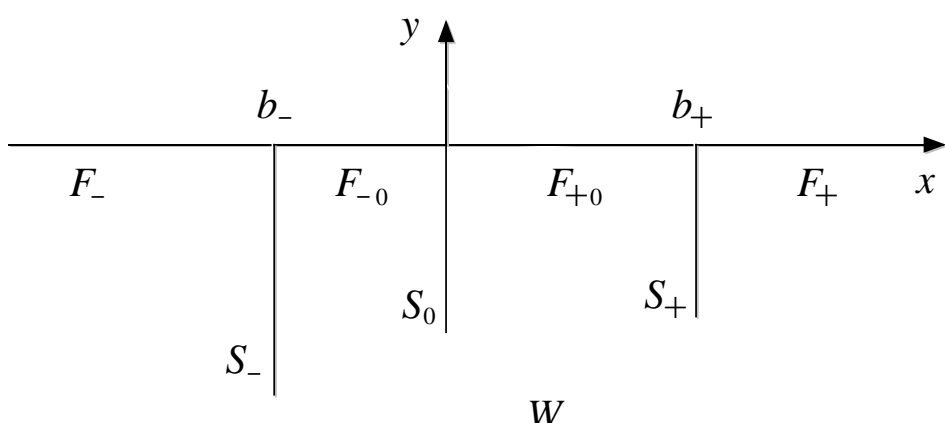

$W$

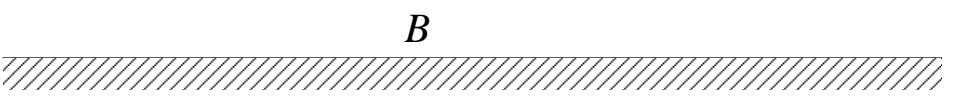

Figure 2: Definition sketch for three-barrier configuration.

the fluid is $F=\left\{x \neq 0, b_{ \pm} ; y=0\right\}=F_{-} \cup F_{-0} \cup F_{+0} \cup F_{+}$, where the portions of the free surface that extend to infinity are $F_{ \pm}=\left\{ \pm x> \pm b_{ \pm} ; y=0\right\}$ and the portions between the barriers are $F_{ \pm 0}=\left\{0< \pm x< \pm b_{ \pm} ; y=0\right\}$. The fluid domain is $W=L \backslash\left\{\bar{S}_{-} \cup \bar{S}_{0} \cup \bar{S}_{+}\right\}$. The boundary conditions on the rigid barriers are

$$
\begin{aligned}
& \phi_{x}\left(b_{+} \pm 0, y\right)=0 \quad \text { on } \quad S_{+}, \\
& \phi_{x}( \pm 0, y)=0 \quad \text { on } \quad S_{0}, \\
& \phi_{x}\left(b_{-} \pm 0, y\right)=0 \quad \text { on } \quad S_{-} .
\end{aligned}
$$

With $w(x)$ defined as in equation (15), the preliminary calculations for the two-barrier configuration apply equally well here. Thus

$$
w_{x x}+\alpha^{2} w=0 \quad \text { on } \quad F_{ \pm} \text {and } \quad F_{ \pm 0}
$$

and it then follows from the radiation condition (8) that $w$ vanishes identically on $F_{ \pm}$. As before, $w_{x}$ is continuous across $x=0, b_{ \pm}$so that

$$
w_{x}\left(b_{-}+0\right)=w_{x}\left(b_{+}-0\right)=0
$$

and

$$
w_{x}(-0)=w_{x}(+0) \text {. }
$$

The solution of (44) satisfying (45) has the form

$$
w(x)=C_{ \pm} \cos \alpha\left(b_{ \pm}-x\right) \quad \text { on } \quad F_{ \pm 0}
$$


where by (46) $C_{-} \sin \alpha b_{-}=C_{+} \sin \alpha b_{+}$. Three cases must be investigated. These are:

$$
\begin{aligned}
& \text { (i) only one of } \sin \alpha b_{-} \text {and } \sin \alpha b_{+} \text {vanishes, } \\
& \text { (ii) } \sin \alpha b_{-}=\sin \alpha b_{+}=0 \\
& \text { (iii) neither } \sin \alpha b_{-} \text {nor } \sin \alpha b_{+} \text {vanishes. }
\end{aligned}
$$

Case (i): Suppose that $\sin \alpha b_{+}=0$ and $\sin \alpha b_{-} \neq 0$ then $C_{-}=0$ and $\alpha b_{+}=m \pi$ for $m=$ $1,2, \ldots$, so that

$$
w(x)=0 \quad \text { on } \quad F_{-0}
$$

and

$$
w(x)=C_{+}(-1)^{m} \cos \alpha x \quad \text { on } \quad F_{+0}
$$

The situation has been reduced to one that is essentially identical to that considered for two barriers in section 3. Thus the three barrier problem has only the trivial solution when $\alpha b_{+}=m \pi$ for $m=1,2, \ldots$, and $\sin \alpha b_{-} \neq 0$; the same is true when the plus and minus signs are interchanged in these last expressions.

Case (ii): Now $\alpha b_{+}=m \pi$ and $\alpha b_{-}=-n \pi$ for $m, n=1,2, \ldots$, so that

$$
w(x)=C_{-}(-1)^{n} \cos \alpha x \quad \text { on } \quad F_{-0}
$$

and

$$
w(x)=C_{+}(-1)^{m} \cos \alpha x \quad \text { on } \quad F_{+0}
$$

and hence (24) is true with $F_{0}$ replaced by $F_{ \pm 0}$. Again, the considerations of section 3 apply and there is only the trivial solution.

Case (iii): Repetition of the considerations for two barriers leads to

$$
K \int_{F_{ \pm 0}}|\phi|^{2} \mathrm{~d} x \leq(1+\lambda) \frac{\ell^{2}+\alpha^{2}}{\sinh ^{2} k h} K \int_{F_{ \pm 0}}|w|^{2} \mathrm{~d} x+\left(1+\lambda^{-1}\right) \frac{\sinh ^{2} k h-K h}{2 \sinh ^{2} k h} \int_{W_{ \pm 0}}\left|\phi_{y}\right|^{2} \mathrm{~d} x \mathrm{~d} y .
$$

However, (24) is not true in general and thus an inequality similar to (39) cannot be derived without the two additional assumptions

$$
\int_{F_{ \pm 0}}|w|^{2} \mathrm{~d} x \leq \alpha^{-2} \int_{F_{ \pm 0}}\left|w_{x}\right|^{2} \mathrm{~d} x
$$

which are equivalent to

$$
\int_{0}^{b_{ \pm}}\left[\cos ^{2} \alpha\left(b_{ \pm}-x\right)-\sin ^{2} \alpha\left(b_{ \pm}-x\right)\right] \mathrm{d} x \leq 0 .
$$


These reduce to the pair of inequalities

$$
\pm \sin 2 \alpha b_{ \pm} \leq 0
$$

which, in particular, contain the case (ii) discussed above. Since these two inequalities guarantee that (39) holds, the proof can be completed in the same way as for two barriers. Thus the following theorem has been obtained.

If the two inequalities (55) hold simultaneously, or when either $\sin \alpha b_{-}$or $\sin \alpha b_{+}$vanishes, then the three-barrier problem defined by equations (1)-(3), (8)-(9), and (43) has only the trivial solution for all $K>\ell$ tanh $\ell h$.

The theorem can be illustrated with the following two simple examples. First of all if $-2 b_{-}=$ $b_{+}=b$ then by (55) the inequalities

$$
\sin 2 \alpha b \leq 0 \quad \text { and } \quad \sin \alpha b \leq 0
$$

must hold simultaneously and the uniqueness theorem holds when

$$
2 \pi m-\frac{\pi}{2} \leq \alpha b \leq 2 \pi m, \quad m=1,2, \ldots
$$

For $-b_{-}=b_{+}=b$, the inequalities $(55)$ reduce to

$$
\sin 2 \alpha b \leq 0
$$

which gives the intervals

$$
\pi m-\frac{\pi}{2} \leq \alpha b \leq \pi m, \quad m=1,2, \ldots
$$

and so there are additional intervals of uniqueness when compared with (57).

If the geometry is symmetric about the $y$ axis so that $-b_{-}=b_{+}=b>0$ and $a_{ \pm}=a>0$ then it is possible to treat separately the cases of symmetric and antisymmetric solutions of (1)-(3), (8)(9), and (43). The symmetric solution $\phi^{(+)}$is defined by $\phi^{(+)}(-x, y)=\phi^{(+)}(x, y)$ so $\phi_{x}^{(+)}(0, y)=0$ for $y \in(-h, 0)$. Hence the fully symmetric case is just a special case of the two-barrier problem in which one barrier, corresponding to the line of symmetry for the three-barrier problem, extends throughout the depth. Thus the following result is established immediately.

For a symmetric arrangement of three-barriers, the homogeneous problem defined by (1)-(3), (8)-(9), and (43) has no symmetric solution other than the trivial solution for $K>\ell \tanh \ell$. 
An antisymmetric solution $\phi^{(-)}$is defined by

$$
\phi^{(-)}(-x, y)=-\phi^{(-)}(x, y)
$$

which implies that

$$
w^{(-)}(x)=\int_{-h}^{0} \phi^{(-)}(x, y) \cosh k(y+h) \mathrm{d} y
$$

is an odd function on $F$. In the same way as above, the solution

$$
w^{(-)}(x)= \pm C \cos \alpha(b \mp x) \quad \text { on } \quad F_{ \pm 0}
$$

is obtained. Since $\phi^{(-)}(0, y)=0$ for $-h<y<-a_{0}$ then, for a non-trivial solution $\phi^{(-)},(62)$ yields

$$
\cos \alpha b=\int_{-a_{0}}^{0} \phi^{(-)}(+0, y) \cosh k(y+h) \mathrm{d} y
$$

( $C$ may be chosen as unity without loss of generality).

No further consequences about uniqueness of $\phi^{(-)}$follow from (62). Thus the following is true.

For a symmetric arrangement of three-barriers, when (59) holds the homogeneous problem defined by (1)-(3), (8)-(9), and (43) has no antisymmetric solution other than the trivial solution for $K>\ell \tanh \ell h$.

For the two-dimensional problem $(\ell=0)$ further progress on antisymmetric solutions of the symmetric three-barrier problem can be made using a stream function. Let $\psi^{(-)}(x, y)$ be a conjugate harmonic function to the antisymmetric velocity potential $\phi^{(-)}(x, y)$. By $(43)$ and the CauchyRiemann equations

$$
\psi^{(-)}(0, y)=0 \quad \text { on } \quad S_{0} \quad \text { and } \quad \psi^{(-)}(b, y)=C \quad \text { on } \quad S_{+},
$$

where $C$ is a real constant and, without loss of generality, the stream function is chosen as zero on $S_{0}$. Differentiation of the definition $(60)$ of $\phi^{(-)}$with respect to $x$ gives

$$
\phi_{x}^{(-)}(-x, y)=\phi_{x}^{(-)}(x, y)
$$

that is the $x$ derivative of $\phi^{(-)}$is an even function in $x$. It follows that

$$
\psi_{y}^{(-)}(-x, y)=\psi_{y}^{(-)}(x, y)
$$


and hence

$$
\psi^{(-)}(-x, y)=\psi^{(-)}(x, y)+F(x)
$$

As $\psi^{(-)}(x, y)$ is harmonic, $F^{\prime \prime}(x)=0$ and so, from (64), $F(x)=A x$ where $A$ is a real constant. Differentiation of (67) with respect to $x$ then yields

$$
\psi_{x}^{(-)}(0, y)=-\frac{1}{2} A
$$

Thus, the function

$$
\Psi^{(-)}(x, y)=\psi^{(-)}(x, y)+\frac{1}{2} A x
$$

is harmonic in $W$ and by (64) and (68) satisfies homogeneous Cauchy data on $S_{0}$. Hence $\Psi^{(-)}$ vanishes identically in $W$ and the stream function

$$
\psi^{(-)}(x, y)=-\frac{1}{2} A x \quad \text { in } \quad W .
$$

This is impossible unless $A=0$ because by the Cauchy-Riemann equations and (10)

$$
\int_{W}\left|\nabla \psi^{(-)}\right|^{2} \mathrm{~d} x \mathrm{~d} y=\int_{W}\left|\nabla \phi^{(-)}\right|^{2} \mathrm{~d} x \mathrm{~d} y<\infty .
$$

As $\psi^{(-)}(x, y)$ vanishes identically in $W, \phi^{(-)}(x, y)$ is a constant in $W$ and from (10) this constant is zero. This, together with the above result on symmetric solutions, yields the following.

In the two-dimensional problem $(\ell=0)$ and for a symmetric arrangement of three-barriers, the homogeneous problem defined by (1)-(3), (8)-(9), and (43) has only the trivial solution for $K>0$.

As for the case of two barriers, the uniqueness results obtained above are true not only when the barriers are surface piercing, but also when they are placed arbitrarily (symmetrically about $x=0$ for $\left.\phi^{( \pm)}\right)$on vertical lines. There must be gaps on at least two of these lines and one of these lines must be $x=0$.

Until the present work, the only uniqueness theorem for three surface-piercing two-dimensional structures was that obtained by Kuznetsov \& Simon (1995b). For $\ell=0$ they proved uniqueness for frequencies below a certain bound depending on the geometry. 


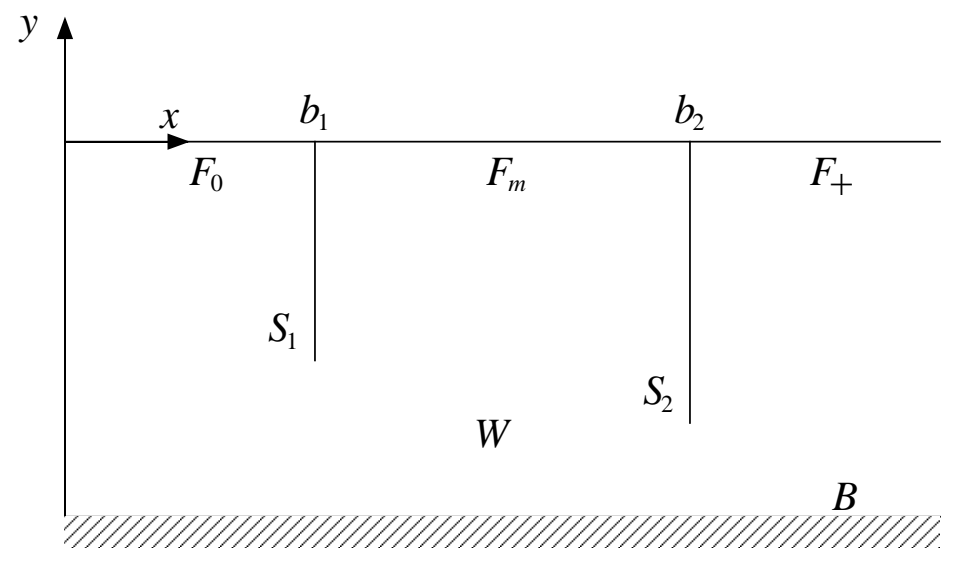

Figure 3: Definition sketch for symmetric four-barrier configuration ( $x \geq 0$ only).

\section{A uniqueness theorem for four barriers}

Consider the four-barrier configuration illustrated in figure 3; the geometry is assumed to be symmetric about $x=0$ so that only $x \geq 0$ need be considered. In $x \geq 0$, two rigid barriers occupy the lines $S_{i}=\left\{x=b_{i} ;-a_{i}<y<0\right\}, i=1,2$, and the free surface of the fluid is $F=F_{0} \cup F_{m} \cup F_{+}$ where $F_{0}=\left\{0 \leq x<b_{1} ; y=0\right\}, F_{m}=\left\{b_{1}<x<b_{2} ; y=0\right\}$, and $F_{+}=\left\{x>b_{2} ; y=0\right\}$. The part of the fluid domain $W_{>}$lying in $x>0$ is $L_{>} \backslash\left\{\bar{S}_{1} \cup \bar{S}_{2}\right\}$ and the boundary conditions on the rigid barriers are

$$
\phi_{x}\left(b_{i} \pm 0, y\right)=0 \quad \text { on } \quad S_{i}, \quad i=1,2 .
$$

Symmetric and antisymmetric solutions, $\phi^{(+)}$and $\phi^{(-)}$respectively, may be considered simultaneously. (The symmetric solutions are just a special case of the three-barrier problem, but for completeness both symmetric and antisymmetric solutions are included in the working below.) Each formula containing ' \pm ' must be considered as two formulae for the two different problems. In a similar way to the two-barrier problem,

$$
w^{( \pm)}(x)=\int_{-h}^{0} \phi^{( \pm)}(x, y) \cosh k(y+h) \mathrm{d} y
$$

satisfies

$$
w_{x x}^{( \pm)}+\alpha^{2} w^{( \pm)}=0 \quad \text { on } \quad F_{0}, F_{m} \quad \text { and } \quad F_{+} .
$$

Again $w_{x}^{( \pm)}$is continuous across the barriers and it is readily found that

$$
w^{( \pm)} \equiv 0 \quad \text { on } \quad F_{+}
$$


and

$$
w^{( \pm)}(x)=C_{ \pm} \cos \alpha\left(b_{2}-x\right) \quad \text { on } \quad F_{m} .
$$

Since

$$
\phi^{( \pm)}(-x, y)= \pm \phi^{( \pm)}(x, y)
$$

then

$$
w^{( \pm)}=D_{ \pm} \cos \left(\alpha x-\frac{\pi}{4} \pm \frac{\pi}{4}\right) \quad \text { on } \quad F_{0} .
$$

Continuity of $w_{x}$ on $x=b_{1}$ then yields

$$
-D_{ \pm} \sin \left(\alpha b_{1}-\frac{\pi}{4} \pm \frac{\pi}{4}\right)=C_{ \pm} \sin \alpha s
$$

where $s=b_{2}-b_{1}$ is the spacing within a barrier pair.

There are four cases to consider:

$$
\begin{aligned}
& \text { (i) } \sin \left(\alpha b_{1}-\frac{\pi}{4} \pm \frac{\pi}{4}\right)=0 \quad \text { and } \quad \sin \alpha s \neq 0 \text {, } \\
& \text { (ii) } \sin \left(\alpha b_{1}-\frac{\pi}{4} \pm \frac{\pi}{4}\right) \neq 0 \text { and } \sin \alpha s=0 \text {, } \\
& \text { (iii) } \sin \left(\alpha b_{1}-\frac{\pi}{4} \pm \frac{\pi}{4}\right)=\sin \alpha s=0, \\
& \text { (iv) } \quad \sin \left(\alpha b_{1}-\frac{\pi}{4} \pm \frac{\pi}{4}\right) \neq 0 \text { and } \quad \sin \alpha s \neq 0
\end{aligned}
$$

Case (i): To be specific it is assumed that $\cos \alpha b_{1}=0$ which corresponds to an antisymmetric solution. Equation (79) gives $C_{-}=0$ and hence $w^{(-)} \equiv 0$ on $F_{m}$ and $F_{+}$. The result obtained for two barriers now applies and it is concluded that the antisymmetric problem has only the trivial solution. The symmetric solution can be treated similarly.

Case (ii): Equation (79) implies that $D_{ \pm}=0$ and an estimate analogous to (29) can be derived for $F_{0}$. Moreover, $(24)$ holds with $w$ and $F_{0}$ replaced by $w^{( \pm)}$and $F_{m}$ respectively and the proof is concluded in the same way as for two barriers.

Case (iii): These conditions ensure that equalities of the form (24) hold on both $F_{m}$ and $F_{0}$ and hence the proof is completed in the same way as for the two-barrier case.

Case (iv): This is treated in the same way as case (iii) for three barriers. Thus, in order to complete the proof, it is assumed that

$$
\int_{b_{1}}^{b_{2}}\left[\cos ^{2} \alpha\left(b_{2}-x\right)-\sin ^{2} \alpha\left(b_{2}-x\right)\right] \mathrm{d} x \leq 0
$$


and

$$
\int_{0}^{b_{1}}\left[\cos ^{2}\left(\alpha x-\frac{\pi}{4} \pm \frac{\pi}{4}\right)-\sin ^{2}\left(\alpha x-\frac{\pi}{4} \pm \frac{\pi}{4}\right)\right] \mathrm{d} x \leq 0
$$

which are equivalent to

$$
\sin 2 \alpha s \leq 0 \quad \text { and } \quad \pm \sin 2 \alpha b_{1} \leq 0 .
$$

The combination of the above four cases results in the following theorem.

If the inequalities (83) hold simultaneously, or if either of (80) cases (i)-(ii) hold, then the solution $\phi^{( \pm)}$of the homogeneous four-barrier problem defined by equations (1)-(3), (8)-(9), and (72) is trivial for $K>\ell \tanh \ell$.

As for the cases of two and three barriers, the uniqueness results obtained above are true not only when the barriers are surface piercing, but also when they are placed arbitrarily on vertical lines and as long as each barrier has at least one gap.

\section{Trapped modes for four barriers}

Here numerical evidence is provided for the existence of trapped modes in the presence of four vertical barriers. The barriers are configured as two symmetrically arranged pairs.

\subsection{Finite depth}

The fluid domain (see figure 3 ) is divided into three regions; these are $0<x<b_{1}, b_{1}<x<b_{2}$ and $x>b_{2}$. In each region the potential is expressed in terms of vertical eigenfunctions that satisfy the free-surface condition (2) and the bed condition (3) and are defined by

$$
\psi_{n}(y)=N_{n}^{-1 / 2} \cos k_{n}(y+h), \quad n=0,1,2, \ldots,
$$

where

$$
N_{n}=\frac{1}{2}\left(1+\frac{\sin 2 k_{n} h}{2 k_{n} h}\right)
$$

The numbers $k_{n}, n=1,2,3, \ldots$, are the positive roots of

$$
K+k_{n} \tanh k_{n} h=0
$$


and $k_{0}=-\mathrm{i} k$, where $k$ is the positive root of (7). In the following the orthogonality relation

$$
\frac{1}{h} \int_{-h}^{0} \psi_{m}(y) \psi_{n}(y) \mathrm{d} y=\delta_{m n}
$$

where $\delta_{m n}$ is the Kronecker delta, will be used extensively.

Expansions appropriate to the three fluid regions are

$$
\begin{gathered}
\phi(x, y)=\sum_{n=0}^{\infty} A_{n} \cosh \alpha_{n} x \psi_{n}(y), \quad 0<x<b_{1}, \\
\phi(x, y)=\sum_{n=0}^{\infty}\left(B_{n} \mathrm{e}^{-\alpha_{n} x}+C_{n} \mathrm{e}^{\alpha_{n} x}\right) \psi_{n}(y), \quad b_{1}<x<b_{2},
\end{gathered}
$$

and

$$
\phi(x, y)=D_{0} E(\alpha x) \psi_{0}(y)+\sum_{n=1}^{\infty} D_{n} \mathrm{e}^{-\alpha_{n}\left(x-b_{2}\right)} \psi_{n}(y), \quad x>b_{2},
$$

where $\alpha_{n}=\sqrt{k_{n}^{2}+\ell^{2}}$ and $\alpha_{0}=-\mathrm{i} \alpha=-\mathrm{i} \sqrt{k^{2}-\ell^{2}}$. Trapped mode solutions have only evanescent terms in $x>b_{2}$. However, the additional term involving

$$
E(\alpha x)=\cos \alpha x+\beta \sin \alpha x
$$

has been included in this region to explore the possibility that other (that is not trapped mode) solutions may be generated by the solution procedure. This term will eventually be set to zero.

\subsubsection{Velocity formulation}

From (88) and (89), the velocity

$$
\phi_{x}\left(b_{1}, y\right) \equiv U^{(1)}(y)=\sum_{n=0}^{\infty} U_{n}^{(1)} \psi_{n}(y)
$$

may be written

$$
U^{(1)}(y)=\sum_{n=0}^{\infty} \alpha_{n} A_{n} \sinh \alpha_{n} b_{1} \psi_{n}(y)=\sum_{n=0}^{\infty} \alpha_{n}\left(-B_{n} \mathrm{e}^{-\alpha_{n} b_{1}}+C_{n} \mathrm{e}^{\alpha_{n} b_{1}}\right) \psi_{n}(y)
$$

and so

$$
U_{n}^{(1)} \equiv \frac{1}{h} \int_{G_{1}} U^{(1)}(y) \psi_{n}(y) \mathrm{d} y=\alpha_{n} A_{n} \sinh \alpha_{n} b_{1}=\alpha_{n}\left(-B_{n} \mathrm{e}^{-\alpha_{n} b_{1}}+C_{n} \mathrm{e}^{\alpha_{n} b_{1}}\right),
$$


where the orthogonality condition (87) has been used and $G_{i}=\left\{x=b_{i} ;-h<y<a_{i}\right\}$ is the gap beneath barrier $i$. Similarly, from (89) and (90), the velocity

$$
\phi_{x}\left(b_{2}, y\right) \equiv U^{(2)}(y)=\sum_{n=0}^{\infty} U_{n}^{(2)} \psi_{n}(y)
$$

may be written

$$
U^{(2)}(y)=\sum_{n=0}^{\infty} \alpha_{n}\left(-B_{n} \mathrm{e}^{-\alpha_{n} b_{2}}+C_{n} \mathrm{e}^{\alpha_{n} b_{2}}\right) \psi_{n}(y)=\alpha D_{0} E^{\prime}\left(\alpha b_{2}\right) \psi_{0}(y)-\sum_{n=1}^{\infty} \alpha_{n} D_{n} \psi_{n}(y)
$$

and so

$$
U_{n}^{(2)} \equiv \frac{1}{h} \int_{G_{2}} U^{(2)}(y) \psi_{n}(y) \mathrm{d} y=\alpha_{n}\left(-B_{n} \mathrm{e}^{-\alpha_{n} b_{2}}+C_{n} \mathrm{e}^{\alpha_{n} b_{2}}\right) \psi_{n}(y)= \begin{cases}\alpha D_{0} E^{\prime}\left(\alpha b_{2}\right), & n=0 \\ -\alpha_{n} D_{n}, & n \neq 0 .\end{cases}
$$

Equations (94) and (97) give, in particular,

$$
B_{n}=-\frac{\mathrm{e}^{\alpha_{n} b_{2}}}{2 \alpha_{n} \sinh \alpha_{n} s}\left(U_{n}^{(1)}-U_{n}^{(2)} \mathrm{e}^{-\alpha_{n} s}\right) \quad \text { and } \quad C_{n}=-\frac{\mathrm{e}^{-\alpha_{n} b_{2}}}{2 \alpha_{n} \sinh \alpha_{n} s}\left(U_{n}^{(1)}-U_{n}^{(2)} \mathrm{e}^{\alpha_{n} s}\right) .
$$

From equations (88)-(90), continuity of the potential (and hence pressure) in the gaps beneath the barriers yields

$$
\sum_{n=0}^{\infty} A_{n} \cosh \alpha_{n} b_{1} \psi_{n}(y)=\sum_{n=0}^{\infty}\left(B_{n} \mathrm{e}^{-\alpha_{n} b_{1}}+C_{n} \mathrm{e}^{\alpha_{n} b_{1}}\right) \psi_{n}(y), \quad y \in G_{1},
$$

and

$$
\sum_{n=0}^{\infty}\left(B_{n} \mathrm{e}^{-\alpha_{n} b_{2}}+C_{n} \mathrm{e}^{\alpha_{n} b_{2}}\right) \psi_{n}(y)=D_{0} E\left(\alpha b_{2}\right) \psi_{0}(y)+\sum_{n=1}^{\infty} D_{n} \psi_{n}(y), \quad y \in G_{2}
$$

Equations (94), (97) and (98) may be used to express all of the coefficients in the last two equations in terms of $U^{(1)}(\cdot)$ and $U^{(2)}(\cdot)$ and this results in the coupled integral equations

$$
\sum_{j=1}^{2} \int_{G_{j}} U^{(j)}(\eta) K^{(i j)}(y, \eta) \mathrm{d} \eta=0, \quad y \in G_{i}(i=1,2),
$$

where

$$
\begin{gathered}
K^{(11)}(y, \eta)=\sum_{n=0}^{\infty} \frac{\sinh \alpha_{n} b_{2} \psi_{n}(y) \psi_{n}(\eta)}{\alpha_{n} h \sinh \alpha_{n} b_{1} \sinh \alpha_{n} s}, \\
K^{(12)}(y, \eta)=K^{(21)}(y, \eta)=-\sum_{n=0}^{\infty} \frac{\psi_{n}(y) \psi_{n}(\eta)}{\alpha_{n} h \sinh \alpha_{n} s},
\end{gathered}
$$


and

$$
K^{(22)}(y, \eta)=-\left(\cot \alpha s+\frac{E\left(\alpha b_{2}\right)}{E^{\prime}\left(\alpha b_{2}\right)}\right) \frac{\psi_{0}(y) \psi_{0}(\eta)}{\alpha h}+\sum_{n=1}^{\infty} \frac{1+\operatorname{coth} \alpha_{n} b_{2}}{\alpha_{n} h} \psi_{n}(y) \psi_{n}(\eta) .
$$

If the scaled velocities

$$
u^{(i)}(y)=U^{(i)}(y) \sin \alpha s, \quad i=1,2,
$$

are introduced and the propagating-wave terms isolated, then the integral equations (101) may be rewritten as

$$
\sum_{j=1}^{2} \int_{G_{j}} u^{(j)}(\eta) K_{1}^{(1 j)}(y, \eta) \mathrm{d} \eta=\frac{U_{0}^{(1)} \sin \alpha b_{2}}{\alpha \sin \alpha b_{1}} \psi_{0}(y)-\frac{U_{0}^{(2)}}{\alpha} \psi_{0}(y), \quad y \in G_{1}(y),
$$

and

$$
\begin{aligned}
\sum_{j=1}^{2} \int_{G_{j}} u^{(j)}(\eta) K_{1}^{(2 j)}(y, \eta) \mathrm{d} \eta=- & \frac{U_{0}^{(1)}}{\alpha} \psi_{0}(y) \\
& +\frac{U_{0}^{(2)}}{\alpha}\left(\cos \alpha s+\frac{E\left(\alpha b_{2}\right)}{E^{\prime}\left(\alpha b_{2}\right)} \sin \alpha s\right) \psi_{0}(y), \quad y \in G_{2}(y)
\end{aligned}
$$

where

$$
\begin{gathered}
K_{1}^{(11)}(y, \eta)=\sum_{n=1}^{\infty} \frac{\sinh \alpha_{n} b_{2} \psi_{n}(y) \psi_{n}(\eta)}{\alpha_{n} h \sinh \alpha_{n} b_{1} \sinh \alpha_{n} s}, \\
K_{1}^{(12)}(y, \eta)=K_{1}^{(21)}(y, \eta)=-\sum_{n=1}^{\infty} \frac{\psi_{n}(y) \psi_{n}(\eta)}{\alpha_{n} h \sinh \alpha_{n} s},
\end{gathered}
$$

and

$$
K_{1}^{(22)}(y, \eta)=\sum_{n=1}^{\infty} \frac{1+\operatorname{coth} \alpha_{n} b_{2}}{\alpha_{n} h} \psi_{n}(y) \psi_{n}(\eta) .
$$

From (97), the terms in $U_{0}^{(2)}$ are zero if either $D_{0}=0$ (that is, there is a trapped mode solution) or if

$$
E^{\prime}\left(\alpha b_{2}\right)=0 \quad \text { and } \quad E^{\prime}\left(\alpha b_{2}\right) \cos \alpha s+E\left(\alpha b_{2}\right) \sin \alpha s=0
$$

that is if $E^{\prime}\left(\alpha b_{2}\right)=E\left(\alpha b_{2}\right) \sin \alpha s=0$. From (91) it is easily seen that $E^{\prime}\left(\alpha b_{2}\right)=E\left(\alpha b_{2}\right)=0$ (which is equivalent to a non-trivial solution for $\phi$ with zero pressure and velocity on a line) is impossible for non-zero $E$. However, the case $E^{\prime}\left(\alpha b_{2}\right)=\sin \alpha s=0$ is possible when $E$ is not 
identically zero. Thus, when looking for trapped mode solutions, it is permissible to set to zero the terms in (106)-(107) involving $U_{0}^{(2)}$, as long as solutions satisfying $\sin \alpha s=0$ are rejected.

As the problem is homogeneous, it is permissible to specify one of the coefficients in (88)-(89). With $A_{0}=1$, the integral equations now reduce to

$$
\sum_{j=1}^{2} \int_{G_{j}} u^{(j)}(\eta) K_{1}^{(1 j)}(y, \eta) \mathrm{d} \eta=-\sin \alpha b_{2} \psi_{0}(y), \quad y \in G_{1}(y),
$$

and

$$
\sum_{j=1}^{2} \int_{G_{j}} u^{(j)}(\eta) K_{1}^{(2 j)}(y, \eta) \mathrm{d} \eta=\sin \alpha b_{1} \psi_{0}(y), \quad y \in G_{2}(y) .
$$

These can be solved for the velocities $u^{(1)}(y)$ and $u^{(2)}(y)$ and trapped mode solutions require the velocities to satisfy the consistency conditions

$$
\int_{G_{1}} u^{(1)}(\eta) \psi_{0}(\eta) \mathrm{d} \eta=-\alpha h \sin \alpha b_{1} \sin \alpha s
$$

and

$$
\int_{G_{2}} u^{(2)}(\eta) \psi_{0}(\eta) \mathrm{d} \eta=0
$$

arising from the specification of $A_{0}$ and $D_{0}$.

Approximate solutions to (112)-(113) are sought in the form

$$
u^{(i)}(y)=\sum_{n=0}^{N_{i}} a_{n}^{(i)} u_{n}^{(i)}(y)
$$

where each $u_{n}^{(i)}(y)$ is chosen to have the correct asymptotic behaviour at the barrier tip (see Porter \& Evans 1995, section 2.1 for details of the functions used). After substitution of this series approximation, multiplication by each of $\left\{u_{m}^{(i)}(y) ; m=0,1, \ldots, N_{i}\right\}$ and integration over $G_{i}$ as appropriate, equations (112)-(113) reduce to

$$
\sum_{j=1}^{2} \sum_{n=0}^{N_{j}} a_{n}^{(j)} K_{m n}^{(1 j)}=-\sin \alpha b_{2} F_{m 0}^{(1)}, \quad m=0,1, \ldots, N_{1},
$$

and

$$
\sum_{j=1}^{2} \sum_{n=0}^{N_{j}} a_{n}^{(j)} K_{m n}^{(2 j)}=\sin \alpha b_{1} F_{m 0}^{(1)}, \quad m=0,1, \ldots, N_{2},
$$


where

$$
\begin{gathered}
K_{m n}^{(11)}=\sum_{r=1}^{\infty} \frac{\sinh \alpha_{r} b_{2} F_{m r}^{(1)} F_{n r}^{(1)}}{\alpha_{r} h \sinh \alpha_{r} s \sinh \alpha_{r} b_{1}}, \\
K_{m n}^{(12)}=K_{n m}^{(21)}=-\sum_{r=1}^{\infty} \frac{F_{m r}^{(1)} F_{n r}^{(2)}}{\alpha_{r} h \sinh \alpha_{r} s}, \\
K_{m n}^{(22)}=\sum_{r=1}^{\infty} \frac{\mathrm{e}^{\alpha_{r} s} F_{m r}^{(2)} F_{n r}^{(2)}}{\alpha_{r} h \sinh \alpha_{r} s}
\end{gathered}
$$

and

$$
F_{m r}^{(i)}=\int_{G_{i}} u_{m}^{(i)}(y) \psi_{r}(y) \mathrm{d} y=N_{n}^{-1 / 2} J_{2 m}\left(k_{n}\left(h-a_{i}\right)\right), \quad i=1,2 .
$$

The consistency conditions (114)-(115) reduce to

$$
\sum_{n=0}^{N_{1}} a_{n}^{(1)} F_{n 0}^{(1)}=-\alpha h \sin \alpha b_{1} \sin \alpha s
$$

and

$$
\sum_{n=0}^{N_{2}} a_{n}^{(2)} F_{n 0}^{(2)}=0
$$

respectively. Trapped mode solutions correspond to a combination of frequency and geometrical parameters such that (123)-(124) are satisfied with $\sin \alpha s \neq 0$, where $\left\{a_{n}^{(i)}, n=1,2, \ldots, N_{i} ; i=\right.$ $1,2\}$ are the solutions of (117)-(118).

The formulation for antisymmetric solutions is similar to the above with $\sinh \alpha_{r} b_{i}$ replaced by $\cosh \alpha_{r} b_{i}$ and $\sin \alpha b_{i}$ replaced by $-\cos \alpha b_{i}$ in the final expressions.

\subsubsection{Pressure-difference formulation}

An alternative formulation may be given in terms of the pressure differences across $x=b_{i}, i=1,2$. For symmetric $\phi$ and $y \in(-h, 0)$ define

$$
\begin{aligned}
P^{(1)}(y) & =\phi\left(b_{1}-0, y\right)-\phi\left(b_{1}+0, y\right) \\
& =\sum_{n=0}^{\infty} A_{n} \cosh \alpha_{n} b_{1} \psi_{n}(y)-\sum_{n=0}^{\infty}\left(B_{n} \mathrm{e}^{-\alpha_{n} b_{1}}+C_{n} \mathrm{e}^{\alpha_{n} b_{1}}\right) \psi_{n}(y)
\end{aligned}
$$


and

$$
\begin{aligned}
P^{(2)}(y) & =\phi\left(b_{2}-0, y\right)-\phi\left(b_{2}+0, y\right) \\
& =\sum_{n=0}^{\infty}\left(B_{n} \mathrm{e}^{-\alpha_{n} b_{2}}+C_{n} \mathrm{e}^{\alpha_{n} b_{2}}\right) \psi_{n}(y)-D_{0} E\left(\alpha b_{2}\right) \psi_{0}(y)-\sum_{n=1}^{\infty} D_{n} \psi_{n}(y) .
\end{aligned}
$$

Multiplication of each of the above by $\psi_{n}(y)$ and integration over the depth gives

$$
P_{n}^{(1)} \equiv \frac{1}{h} \int_{S_{1}} P^{(1)}(y) \psi_{n}(y)=A_{n} \cosh \alpha_{n} b_{1}-\left(B_{n} \mathrm{e}^{-\alpha_{n} b_{1}}+C_{n} \mathrm{e}^{\alpha_{n} b_{1}}\right)
$$

and

$$
P_{n}^{(2)} \equiv \frac{1}{h} \int_{S_{2}} P^{(2)}(y) \psi_{n}(y)= \begin{cases}B_{0} \mathrm{e}^{i \alpha b_{2}}+C_{0} \mathrm{e}^{-i \alpha b_{2}}-D_{0} E\left(\alpha b_{2}\right), & n=0, \\ B_{n} \mathrm{e}^{-\alpha_{n} b_{2}}+C_{n} \mathrm{e}^{\alpha_{n} b_{2}}-D_{n}, & n \neq 0 .\end{cases}
$$

These last two equations, together with equations (94), (97) and (98), allow all of the coefficients in the eigenfunction expansions to be expressed in terms of $P_{n}^{(1)}$ and $P_{n}^{(2)}$. In particular,

$$
U_{n}^{(1)}=\alpha_{n} \sinh \alpha_{n} b_{1} \mathrm{e}^{-\alpha_{n} b_{1}}\left(P_{n}^{(1)}+\mathrm{e}^{-\alpha_{n} s} P_{n}^{(2)}\right)
$$

and

$$
U_{n}^{(2)}= \begin{cases}\left(P_{0}^{(1)} \sin \alpha b_{1}+P_{0}^{(2)} \sin \alpha b_{2}\right) /\left(\sin \alpha b_{2} V(\alpha)\right), & n=0, \\ \alpha_{n} \mathrm{e}^{-\alpha_{n} b_{2}}\left(P_{n}^{(1)} \sinh \alpha_{n} b_{1}+P_{n}^{(2)} \sinh \alpha_{n} b_{2}\right), & n \neq 0,\end{cases}
$$

where

$$
V(\alpha)=\frac{1}{\alpha \sin \alpha s}\left(\frac{\sin \alpha b_{1}}{\sin \alpha b_{2}}-\cos \alpha s-\frac{E\left(\alpha b_{2}\right)}{E^{\prime}\left(\alpha b_{2}\right)} \sin \alpha s\right) .
$$

Now

$$
U^{(i)}(y)=\sum_{n=0}^{\infty} U_{n}^{(i)} \psi_{n}(y)=0, \quad y \in S_{i}
$$

and thus from (129)-(130)

$$
\begin{aligned}
{\left[P_{0}^{(1)}(\cos \alpha s\right.} & \left.\left.+\frac{E\left(\alpha b_{2}\right)}{E^{\prime}\left(\alpha b_{2}\right)} \sin \alpha s\right)+P_{0}^{(2)}\right] \frac{\sin \alpha b_{1} \psi_{0}(y)}{\sin \alpha b_{2} V(\alpha)} \\
& +\sum_{n=1}^{\infty} \alpha_{n} \sinh \alpha b_{1} \mathrm{e}^{-\alpha_{n} b_{1}}\left(P_{n}^{(1)}+\mathrm{e}^{-\alpha_{n} s} P_{n}^{(2)}\right) \psi_{n}(y)=0, \quad y \in S_{1},
\end{aligned}
$$


and

$$
\begin{aligned}
& \left(P_{0}^{(1)} \frac{\sin \alpha b_{1}}{\sin \alpha b_{2}}+P_{0}^{(2)}\right) \frac{\psi_{0}(y)}{V(\alpha)} \\
& \quad+\sum_{n=1}^{\infty} \alpha_{n} \mathrm{e}^{-\alpha_{n} b_{2}}\left(\sinh \alpha_{n} b_{1} P_{n}^{(1)}+P_{n}^{(2)} \sinh \alpha_{n} b_{2}\right) \psi_{n}(y)=0, \quad y \in S_{2}
\end{aligned}
$$

To obtain a trapped mode solution it is required that $D_{0}=0$. From (97) and (130),

$$
P_{0}^{(2)}=-\frac{\sin \alpha b_{1}}{\sin \alpha b_{2}} P_{0}^{(1)}
$$

implies $D_{0}=0$ unless

$$
\alpha E^{\prime}\left(\alpha b_{2}\right) V(\alpha)=-\left(E^{\prime}\left(\alpha b_{2}\right) \cot \alpha b_{2}+E\left(\alpha b_{2}\right)\right)=0 .
$$

From (91), (136) is satisfied by taking $\beta=0$; that is it appears that the choice (135) need not necessarily correspond to a trapped mode. However, we also have the consistency conditions

$$
\frac{1}{h} \int_{S_{1}} P^{(1)}(y) \psi_{0}(y) \mathrm{d} t=-\frac{U_{0}^{(1)}}{\alpha}\left(\cot \alpha s+\cot \alpha b_{1}\right)+\frac{U_{0}^{(2)}}{\alpha \sin \alpha s}
$$

and

$$
\frac{1}{h} \int_{S_{2}} P^{(2)}(y) \psi_{0}(y) \mathrm{d} t=\frac{U_{0}^{(1)}}{\alpha \sin \alpha s}-\frac{U_{0}^{(2)}}{\alpha}\left(\cot \alpha s+\frac{E\left(\alpha b_{2}\right)}{E^{\prime}\left(\alpha b_{2}\right)}\right) .
$$

The terms in $U_{0}^{(2)}$ are zero if either $D_{0}=0$ or

$$
E^{\prime}\left(\alpha b_{2}\right)=0 \quad \text { and } \quad E^{\prime}\left(\alpha b_{2}\right) \cot \alpha s+E\left(\alpha b_{2}\right)=0
$$

which are the same conditions that arise in the velocity formulation. Hence, solutions obtained from using (135) correspond to trapped modes unless $\sin \alpha s=0$.

With (135), so that $U_{0}^{(2)}=0$, and the choice $U_{0}^{(1)}=-\alpha \sin \alpha b_{1}$ made previously, the above may be simplified to

$$
\sum_{n=1}^{\infty} \alpha_{n} h \sinh \alpha b_{1} \mathrm{e}^{-\alpha_{n} b_{1}}\left(\int_{S_{1}} p^{(1)}(t) \psi_{n}(t) \mathrm{d} t+\mathrm{e}^{-\alpha_{n} s} \int_{S_{2}} p^{(2)}(t) \psi_{n}(t) \mathrm{d} t\right) \psi_{n}(y)=\psi_{0}(y), \quad y \in S_{1},
$$

and

$$
\sum_{n=1}^{\infty} \alpha_{n} h \mathrm{e}^{-\alpha_{n} b_{2}}\left(\sinh \alpha_{n} b_{1} \int_{S_{1}} p^{(1)}(t) \psi_{n}(t) \mathrm{d} t+\sinh \alpha_{n} b_{2} \int_{S_{2}} p^{(2)}(t) \psi_{n}(t) \mathrm{d} t\right) \psi_{n}(y)=0, \quad y \in S_{2}
$$


with the consistency conditions

$$
\int_{S_{1}} p^{(1)}(t) \psi_{0}(t) \mathrm{d} t=\frac{\sin \alpha a_{2}}{\alpha h \sin \alpha a_{1} \sin \alpha s}
$$

and

$$
\int_{S_{2}} p^{(2)}(t) \psi_{0}(t) \mathrm{d} t=-\frac{1}{\alpha h \sin \alpha s}
$$

here the substitution

$$
P^{(i)}(y)=\alpha h^{2} \sin \alpha a_{1} p^{(i)}(y), \quad i=1,2,
$$

has been made. The integrations and summations in (140)-(141) cannot be reversed as the resulting infinite series are divergent.

Solutions to (140)-(141) are sought in the form

$$
p^{(i)}(y)=\sum_{n=0}^{N_{i}} b_{n}^{(i)} p_{n}^{(i)}(y)
$$

where each $p_{n}^{(i)}(y)$ has the correct asymptotic behaviour at the barrier tip (see Porter \& Evans 1995, section 2.1 for details of the functions used). After substitution of this series approximation, multiplication by each of $\left\{p_{m}^{(i)}(y) ; m=0,1, \ldots, N_{i}\right\}$ and integration over $S_{i}$ as appropriate, equations (140)-(141) reduce to

$$
\sum_{j=1}^{2} \sum_{n=0}^{N_{j}} b_{n}^{(j)} M_{m n}^{(1 j)}=G_{m 0}^{(1)}, \quad m=0,1, \ldots, N_{1}
$$

and

$$
\sum_{j=1}^{2} \sum_{n=0}^{N_{j}} b_{n}^{(j)} M_{m n}^{(2 j)}=0, \quad m=0,1, \ldots, N_{2},
$$

where

$$
\begin{gathered}
M_{m n}^{(11)}=\sum_{r=1}^{\infty} \alpha_{r} h \sinh \alpha_{r} b_{1} \mathrm{e}^{-\alpha_{r} b_{1}} G_{m r}^{(1)} G_{n r}^{(1)}, \\
M_{m n}^{(12)}=M_{n m}^{(21)}=\sum_{r=1}^{\infty} \alpha_{r} h \sinh \alpha_{r} b_{1} \mathrm{e}^{-\alpha_{r} b_{2}} G_{m r}^{(1)} G_{n r}^{(2)}, \\
M_{m n}^{(22)}=\sum_{r=1}^{\infty} \alpha_{r} h \sinh \alpha_{r} b_{2} \mathrm{e}^{-\alpha_{r} b_{2}} G_{m r}^{(2)} G_{n r}^{(2)},
\end{gathered}
$$


and

$$
G_{m r}^{(i)}=\int_{S_{i}} p_{m}^{(i)}(y) \psi_{r}(y) \mathrm{d} y=N_{n}^{-1 / 2} \frac{\cos k_{n} h}{k_{n} h} J_{2 m+1}\left(k_{n} a_{i}\right), \quad i=1,2 .
$$

The consistency conditions (142)-(143) reduce to

$$
\sum_{n=0}^{N_{1}} b_{n}^{(1)} G_{n 0}^{(1)}=\frac{\sin \alpha b_{2}}{\alpha h \sin \alpha b_{1} \sin \alpha s}
$$

and

$$
\sum_{n=0}^{N_{2}} b_{n}^{(2)} G_{n 0}^{(2)}=-\frac{1}{\alpha h \sin \alpha s}
$$

respectively. Trapped mode solutions correspond to a combination of frequency and geometrical parameters such that $(152)-(153)$ are satisfied with $\sin \alpha s \neq 0$, where $\left\{b_{n}^{(i)}, n=1,2, \ldots, N_{i} ; i=\right.$ $1,2\}$ are the solutions of (146)-(147).

The formulation for antisymmetric solutions is similar to the above with $\sinh \alpha_{r} b_{i}$ replaced by $\cosh \alpha_{r} b_{i}$ and $\sin \alpha b_{i}$ replaced by $-\cos \alpha b_{i}$ in the final expressions.

\subsection{Infinite depth}

An alternative approach to the interaction of water waves with thin plates is to introduce an appropriate Green's function and formulate the problem as an integral equation, which due to the nature of the geometry, turns out to be hypersingular, involving a Hadamard finite-part integral. This method has been pioneered in a series of papers by Parsons \& Martin (1992, 1994, 1995) and has been used recently by Linton \& Kuznetsov (1997) to investigate the existence of trapped modes in the presence of a pair of symmetrically placed inclined barriers. The Green's function has a much simpler form in infinite depth and we will restrict attention to the deep-water case in this section. Furthermore the Green's function takes on a different form depending on whether $\ell$ is zero or not. Here we will consider the case $\ell=0$, but the necessary formulae for the Green's function when $\ell>0$ can be found in Parsons \& Martin (1995).

The formulation of the problem for the symmetric four-barrier problem illustrated in figure 3 is very similar to that given in Linton \& Kuznetsov (1997). We obtain a hypersingular integral equation for the unknown discontinuity in the potential across the plates, $[\phi]$. The result is

$$
\oint_{S}[\phi(q)] \frac{\partial^{2} G(p, q)}{\partial n_{p} \partial n_{q}} \mathrm{~d} s_{q}=0, \quad p \in S,
$$


where $p$ and $q$ are points on $S=S_{1} \cup S_{2}$, the integral is a Hadamard finite-part integral and $G(P, Q)$ is the Green's function which is chosen to satisfy

$$
\begin{aligned}
& \nabla^{2} G=0 \quad \text { in the fluid, } P \neq Q \\
& G \sim \ln |P-Q| \quad \text { as } P \rightarrow Q, \\
& K G-\frac{\partial G}{\partial y}=0 \quad \text { on } y=0, \\
& \frac{\partial G}{\partial x}=0 \quad \text { on } x=0, \\
& \frac{\partial G}{\partial x}-\mathrm{i} K G=o(1) \quad \text { as } x \rightarrow \infty .
\end{aligned}
$$

With this $G$, equation (154) will have a non-trivial solution for a given value of $K$ if and only if a trapped mode exists at that frequency.

With $P=(x, y)$ and $Q=(\xi, \eta)$ we obtain for the solution to (155)-(159),

$$
G=\frac{1}{2}\left[\ln \frac{X^{2}+Y^{2}}{X^{2}+\tilde{Y}^{2}}+\ln \frac{\tilde{X}^{2}+Y^{2}}{\tilde{X}^{2}+\tilde{Y}^{2}}\right]-2\left[\phi_{0}(X, \tilde{Y})+\phi_{0}(\tilde{X}, \tilde{Y})\right] .
$$

Here $X=x-\xi, \tilde{X}=x+\xi, Y=y-\eta, \tilde{Y}=y+\eta$, and

$$
\phi_{0}(X, Y)=\int_{0}^{\infty} \frac{\mathrm{e}^{k Y} \cos k X}{k-K} \mathrm{~d} k
$$

where the integration contour is indented so as to pass below the pole at $k=K$. The function $\phi_{0}$ is easily evaluated using an expansion derived by Yu \& Ursell (1961). For vertical barriers $\mathbf{n}_{p}=\mathbf{n}_{q}=(-1,0)$, and it can be shown that

$$
\begin{aligned}
\frac{\partial^{2} G}{\partial n_{p} \partial n_{q}}= & -\frac{1}{X^{2}+Y^{2}}+\frac{1-2 K \tilde{Y}}{\tilde{X}^{2}+\tilde{Y}^{2}}+\frac{X^{2}-\tilde{Y}^{2}}{\left(X^{2}+\tilde{Y}^{2}\right)^{2}}-\frac{\tilde{X}^{2}-Y^{2}}{\left(\tilde{X}^{2}+Y^{2}\right)^{2}}+\frac{2 K \tilde{Y}}{X^{2}+\tilde{Y}^{2}} \\
& +\frac{2 X^{2}}{\left(X^{2}+Y^{2}\right)^{2}}-\frac{2 \tilde{X}^{2}}{\left(\tilde{X}^{2}+\tilde{Y}^{2}\right)^{2}}+2 K^{2}\left[\phi_{0}(\tilde{X}, \tilde{Y})-\phi_{0}(X, \tilde{Y})\right] .
\end{aligned}
$$

A suitable parametrization for $q \equiv(\xi, \eta) \in S_{i}, i=1,2$ is given by

$$
\xi=b_{i}, \quad \eta\left(t_{i}\right)=-a_{i} t_{i}, \quad 0 \leq t_{i} \leq 1,
$$

and $p \equiv(x, y) \in S_{i}$ is parametrized in the same way but with $t_{i}$ replaced by $s_{i}$. We introduce the functions $f_{i}(t) \equiv[\phi(q(t))]$ on $S_{i}$ and approximate them as

$$
f_{i}(t) \approx\left(1-t_{i}^{2}\right)^{1 / 2} \sum_{n=1}^{N_{i}} a_{n}^{(i)} U_{n-1}\left(t_{i}\right)
$$


where $U_{n}(\cdot)$ is a Chebyshev polynomial of the second kind and $a_{n}^{(i)}, n=1, \ldots, N_{i}$ are unknown constants. This form for $f_{i}(t)$ ensures the correct behaviour at the ends of the plate (see Martin 1991).

Substituting (164), together with the given parameterization of $p$ and $q$, into (154) leads to

$$
\sum_{j=1}^{2} \sum_{n=1}^{N_{j}} a_{n}^{(j)} \int_{S_{j}} g_{n}\left(t_{j}\right) H\left(s_{i}, t_{j}\right) \mathrm{d} t_{j}=0, \quad 0 \leq s_{i} \leq 1, \quad i=1,2,
$$

where $g_{n}(t)=\left(1-t^{2}\right)^{1 / 2} U_{n-1}(t)=\sin \left(n \cos ^{-1} t\right)$ and $H(s, t)$ is the parametrized form of $\partial^{2} G / \partial n_{p} \partial n_{q}$.

Equation (165) is converted into a finite system of equations by collocating at the points

$$
s_{m}^{(i)}=\frac{1}{2}\left\{1+\cos \left[\frac{(2 m-1) \pi}{2 N_{i}}\right]\right\}, \quad m=1, \ldots, N_{i} .
$$

We thus obtain the $\left(N_{1}+N_{2}\right) \times\left(N_{1}+N_{2}\right)$ linear system for the unknowns $a_{n}^{(1)}$ and $a_{n}^{(2)}$ :

$$
\sum_{j=1}^{2} \sum_{n=1}^{N_{j}} A_{m n}^{i j} a_{n}^{j}=0, \quad m=1, \ldots, N_{i}, \quad i=1,2,
$$

where

$$
A_{m n}^{i j}=\int_{S_{j}} g_{n}\left(t_{j}\right) H\left(s_{m}^{(i)}, t_{j}\right) \mathrm{d} t_{j}
$$

When $i \neq j$ the evaluation of $A_{m n}^{i j}$ is straightforward, if lengthy. But when $i=j$ special care must be taken due to the hypersingular nature of the integral. In this latter case we have $X=0$, $\tilde{X}=2 b_{i}, Y=-a_{i}\left(t_{i}-s_{m}^{(i)}\right), \tilde{Y}=-a_{i}\left(t_{i}+s_{m}^{(i)}\right)$ and so, from (162),

$$
\begin{aligned}
H\left(s_{m}^{(i)}, t_{i}\right) & =-\frac{1}{a_{i}^{2}\left(t_{i}-s_{m}^{(i)}\right)^{2}}+\frac{1+2 K a_{i}\left(t_{i}+s_{m}^{(i)}\right)}{4 b_{i}^{2}+a_{i}^{2}\left(t_{i}+s_{m}^{(i)}\right)^{2}}-\frac{1}{a_{i}^{2}\left(t_{i}+s_{m}^{(i)}\right)^{2}} \\
& -\frac{4 b_{i}^{2}-a_{i}^{2}\left(t_{i}-s_{m}^{(i)}\right)^{2}}{\left[4 b_{i}^{2}+a_{i}^{2}\left(t_{i}-s_{m}^{(i)}\right)^{2}\right]^{2}}-\frac{2 K}{a_{i}\left(t_{i}+s_{m}^{(i)}\right)}-\frac{8 b_{i}^{2}}{\left[4 b_{i}^{2}+a_{i}^{2}\left(t_{i}+s_{m}^{(i)}\right)^{2}\right]^{2}} \\
& +2 K^{2}\left[\phi_{0}\left(2 b_{i}, a_{i}\left(t_{i}+s_{m}^{(i)}\right)\right)-\phi_{0}\left(0, a_{i}\left(t_{i}+s_{m}^{(i)}\right)\right)\right] .
\end{aligned}
$$

The only troublesome term is the first one, which leads to a contribution

$$
-f_{0}^{1} \frac{g_{n}(t)}{a_{i}^{2}\left(t-s_{m}^{(i)}\right)^{2}} \mathrm{~d} t
$$

This integral can be computed simply using recurrence relations listed in Parsons \& Martin (1994).

Our objective is to find values of $K$ for which, in the limit as $N_{1}, N_{2} \rightarrow \infty$, non-trivial solutions to the system of equations (167) exist. In other words we must find values of $K$ at which the 
determinant of the matrix whose $(m, n)$-th element is

$$
\begin{array}{ll}
A_{m n}^{11} & \text { if } 1 \leq m \leq N_{1} \text { and } 1 \leq n \leq N_{1} \\
A_{m n}^{12} & \text { if } 1 \leq m \leq N_{1} \text { and } N_{1}+1 \leq n \leq N_{1}+N_{2}, \\
A_{m n}^{21} & \text { if } N_{1}+1 \leq m \leq N_{1}+N_{2} \text { and } 1 \leq n \leq N_{1} \\
A_{m n}^{22} & \text { if } N_{1}+1 \leq m \leq N_{1}+N_{2} \text { and } N_{1}+1 \leq n \leq N_{1}+N_{2},
\end{array}
$$

is zero. The method used to locate such zeros is described in Linton \& Kuznetsov (1997).

The formulation for antisymmetric solutions is very similar. Equation (158) is replaced by $G=0$ on $x=0$ and the correct form for $G$ is now

$$
G=\frac{1}{2}\left[\ln \frac{X^{2}+Y^{2}}{X^{2}+\tilde{Y}^{2}}-\ln \frac{\tilde{X}^{2}+Y^{2}}{\tilde{X}^{2}+\tilde{Y}^{2}}\right]-2\left[\phi_{0}(X, \tilde{Y})-\phi_{0}(\tilde{X}, \tilde{Y})\right] .
$$

\subsection{Results}

For finite water depth, trapped modes may be identified through the velocity formulation of section 6.1.1 or the pressure-difference formulation of section 6.1.2. In the velocity formulation, trapped modes are identified by searching for points in the parameter space at which both of equations (123) and (124) are satisfied, where $\left\{a_{n}^{(i)}, n=1,2, \ldots, N_{i} ; i=1,2\right\}$ are solutions of equations (117) and (118). For a fixed barrier pair configuration (that is for fixed $a_{1} / h, a_{2} / h$ and $s / h$ ) the satisfaction of (123) and (124) each corresponds to a line in the $k$ - $b_{1}$ plane. These lines are straightforward to compute; other than intersections at $\sin \alpha s=0$, each intersection point of these lines corresponds to a trapped mode. An alternative solution procedure would be to reduce equations (101) to a homogeneous algebraic system and look for zeros of the corresponding determinant. However, such zeros will be isolated points in the parameter space and hence difficult to locate; for this reason the solution procedure outlined above is preferred here. In the pressure-difference formulation, trapped modes are identified by searching for points in the parameter space at which both of equations (152) and (153) are satisfied, where $\left\{b_{n}^{(i)}, n=1,2, \ldots, N_{i} ; i=1,2\right\}$ are solutions of equations (146) and (147). Solutions of (117)-(118) are easier to locate than solutions of (146)-(147) because lines in the parameter space corresponding to (146)-(147) have regions of very rapid change. Thus, the calculations reported here were made with the velocity formulation although all numerical values given were checked with the pressure difference formulation and agreement was obtained to at least the number of digits shown in the tables below. 
The infinite summations appearing in the definitions of the $K_{m n}^{(i j)}$ and $M_{m n}^{(i j)}$ (see equations 117118 and 146-147) are evaluated using the technique described by Porter \& Evans (1995, section 5). Thus, for example, suppose that a typical summation is

$$
T_{m n}=\sum_{r=1}^{\infty} \mathcal{T}_{m n, r}
$$

then this is evaluated as

$$
T_{m n} \approx \sum_{r=1}^{M_{1}} \mathcal{T}_{m n, r}+C(-1)^{(m+n)} \sum_{r=M_{1}}^{M_{1}+M_{2}} \mathcal{S}_{r}
$$

where $C(-1)^{(m+n)} \mathcal{S}_{r}$ is the leading-order asymptotic form of $\mathcal{T}_{m n, r}$ as $r \rightarrow \infty$. It turns out for each of $K_{m n}^{(i j)}$ and $M_{m n}^{(i j)}, C$ is a constant that depends only on the geometry. Moreover, $\mathcal{S}_{r}$ is independent of $\alpha, m$ and $n$ and is the same for all $K_{m n}^{(i j)}$ and $M_{m n}^{(i j)}$, and so need only be evaluated once for a particular geometry.

The basic strategy adopted in the numerical calculations was to fix the geometry of one barrier pair, that is fix $a_{1} / h, a_{2} / h$ and $s / h$, and then vary the wavenumber parameter $k h$ and spacing between barriers $b_{1} / h$ to obtain trapped-mode solutions. For fixed truncation levels, the accuracy of the calculations depends significantly on $k h$ and $a_{i} / h$. However, for the calculations reported here the values $N_{1}=N_{2}=20, M_{1}=8000$ and $M_{2}=5 \times 10^{6}$ are sufficient to obtain six-figure agreement between the velocity and pressure-difference formulation.

The heuristic argument of Evans \& Porter (1998), reproduced in the introduction of the present paper, suggests that trapped waves for four barriers will occur at frequencies close to those at which there are zeros of transmission for a pair of barriers. This wide spacing argument can then be used to estimate the required spacing between pairs of barriers; this was done by Linton \& Kuznetsov (1997) when looking for trapped modes in the presence of one pair of inclined barriers. The wide-spacing argument proceeds as follows. Let $x=B=\left(b_{1}+b_{2}\right) / 2$ be the mid line between the pair of barriers in $x>0$, and let $\alpha=\alpha_{T}$ be the $x$ component of the wavenumber corresponding to a zero of transmission for that barrier pair. At this zero the reflection coefficient $R=\mathrm{e}^{\mathrm{i} \chi}$ for some real phase $\chi$. Between the pairs of barriers there will be waves propagating to right and left which will combine to form a standing wave. For symmetric modes this will happen if

$$
\mathrm{e}^{\mathrm{i} \alpha_{T}(x-B)}+R \mathrm{e}^{-\mathrm{i} \alpha_{T}(x-B)}=A \cos \alpha_{T} x
$$

which is equivalent to

$$
\mathrm{e}^{\mathrm{i}\left(\chi+2 \alpha_{T} B\right)}=1
$$




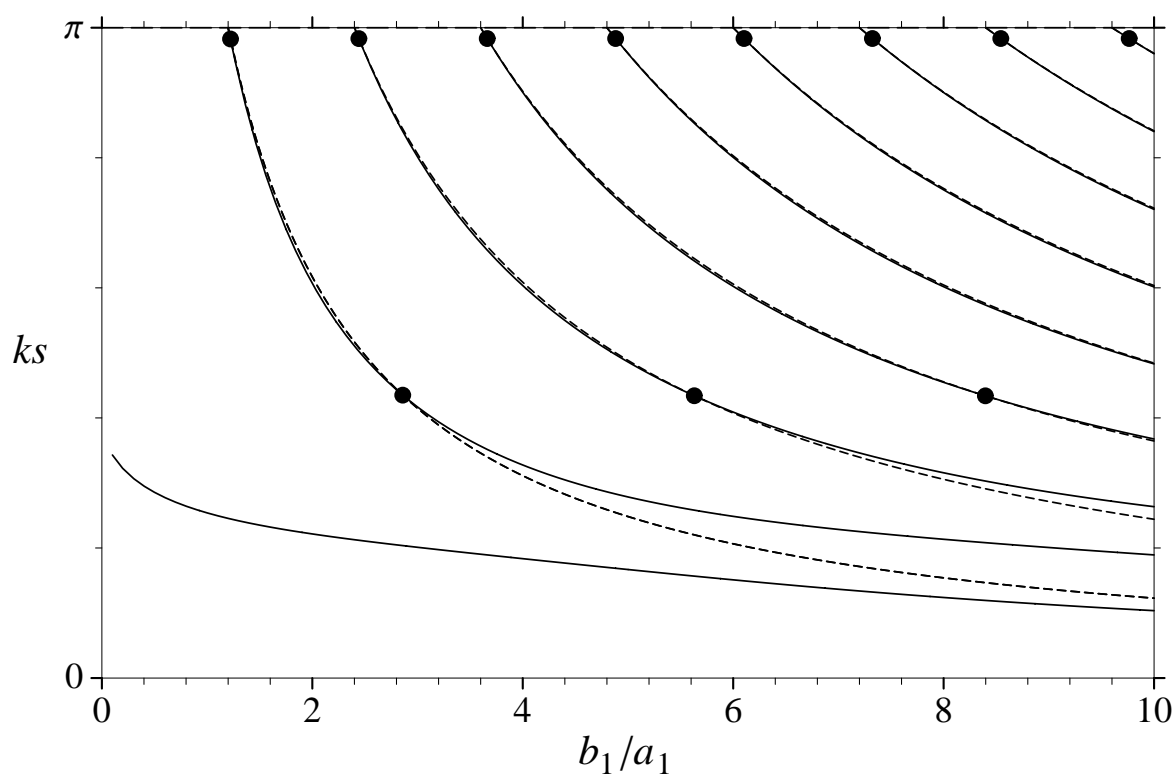

Figure 4: Lines in the $k s-b_{1} / a$ plane where velocity conditions are satisfied; equation (123), - - - equation (124). Intersection points (trapped modes) are marked •. Here $\ell h=0, a_{1} / h=a_{2} / h=0.1, s / h=0.12$.

so that the spacing

$$
B=\frac{2 n \pi-\chi}{2 \alpha_{T}}
$$

for some integer $n$. For antisymmetric modes

$$
\mathrm{e}^{\mathrm{i} \alpha_{T}(x-B)}+R \mathrm{e}^{-\mathrm{i} \alpha_{T}(x-B)}=A \sin \alpha_{T} x
$$

which gives a spacing

$$
B=\frac{(2 n-1) \pi-\chi}{2 \alpha_{T}}
$$

for some integer $n$.

Results are presented first for the case $\ell h=0, a_{1} / h=a_{2} / h=0.1$. These parameters correspond to the results of Porter \& Evans (1995) for zeros of transmission for a barrier pair presented in their figure 8. Shown in figure 4 for the spacing $s / h=0.12$ are the lines in the $k s-b_{1} / a_{1}$ plane where equations (123)-(124) are satisfied; these equations correspond to symmetric solutions. The lines have a number of intersection points other than the precluded intersections at $k s=\pi$, thus confirming the existence of trapped modes. For the value of $s / h$ used here, it can be seen from Porter \& Evans (1995, figure 8) that there are two zeros of transmission for $0<k s<\pi$; these zeros 


\begin{tabular}{|c|c|c|c|c|c|c|c|}
\hline \multicolumn{4}{|c|}{ Finite depth } & \multicolumn{4}{c|}{ Infinite depth } \\
\hline Wide spacing & \multicolumn{2}{|c|}{ Exact } & \multicolumn{2}{c|}{ Wide spacing } & \multicolumn{2}{c|}{ Exact } \\
\hline$b_{1} / a_{1}$ & $K a_{1}$ & $b_{1} / a_{1}$ & $K a_{1}$ & $b_{1} / a_{1}$ & $K a_{1}$ & $b_{1} / a_{1}$ & $K a_{1}$ \\
\hline 2.8672 & 1.1361 & 2.8592 & 1.1388 & 2.8677 & 1.1359 & 2.8590 & 1.1389 \\
5.6326 & 1.1361 & 5.6304 & 1.1365 & 5.6335 & 1.1359 & 5.6298 & 1.1366 \\
8.3979 & 1.1361 & 8.3969 & 1.1362 & - & - & - & - \\
\hline 1.2217 & 2.5745 & 1.2226 & 2.5726 & 1.2217 & 2.5746 & 1.2211 & 2.5757 \\
2.4420 & 2.5745 & 2.4423 & 2.5742 & 2.4419 & 2.5746 & 2.4420 & 2.5745 \\
3.6622 & 2.5745 & 3.6623 & 2.5745 & - & - & - & - \\
\hline
\end{tabular}

Table 1: A selection of symmetric trapped modes. For all calculations, $\ell s=0$, $s / a_{1}=s / a_{2}=1.2$. For the finite depth calculations $a_{1} / h=0.1$.

occur at $k h=11.361,25.745$ or, equivalently, $k s=0.434 \pi, 0.983 \pi$. It is apparent from figure 4 that there is a close association between these values of $k s$ and those at which trapped modes occur. Further evidence of this for both symmetric and antisymmetric modes is given in tables 1 and 2 where a variety of numerically calculated trapped-mode spacing-frequency pairs are compared with the corresponding predictions of the wide-spacing formulas (175) and (177). Additional evidence is provided by calculations for infinite depth made using the method described in section 6.2. Apart from the depth, the parameters for the finite and infinite depth calculations are the same. The finite depth calculations are made for a small barrier submergence compared to the depth and hence there is good agreement between the finite- and infinite-depth calculations. The blanks in the table for infinite depth correspond to large $K b_{1}$ when it is difficult to compute trapped modes using the method of section 6.2 because some of the integrals defined by (168) become highly oscillatory.

The question arises "Are trapped modes always associated with zeros of transmission"? The numerical evidence suggests that this is indeed the case. It is apparent from Porter \& Evans (1995, figure 8) that as the spacing $s$ within a barrier pair increases then a given zero of transmission is lost. The present figure 5 shows the lines where the individual velocity conditions are satisfied for a spacing for which there are no zeros of transmission within the illustrated range of $k s$; the lines do not intersect and so there are no trapped modes. Figure 8 of Porter \& Evans (1995) also indicates that there are no zeros of transmission for $\pi<k s<2 \pi$; numerical investigations with a number of geometries have revealed no trapped modes for this range of $k s$. For sufficiently small spacing, there 


\begin{tabular}{|c|c|c|c|c|c|c|c|}
\hline \multicolumn{4}{|c|}{ Finite depth } & \multicolumn{4}{c|}{ Infinite depth } \\
\hline \multicolumn{2}{|c|}{ Wide spacing } & \multicolumn{2}{|c|}{ Exact } & \multicolumn{2}{c|}{ Wide spacing } & \multicolumn{2}{c|}{ Exact } \\
\hline$b_{1} / a_{1}$ & $K a_{1}$ & $b_{1} / a_{1}$ & $K a_{1}$ & $b_{1} / a_{1}$ & $K a_{1}$ & $b_{1} / a_{1}$ & $K a_{1}$ \\
\hline 1.4846 & 1.1361 & 1.5060 & 1.1239 & 1.4849 & 1.1361 & 1.4997 & 1.1277 \\
4.2499 & 1.1361 & 4.2536 & 1.1352 & 4.2506 & 1.1361 & 4.2536 & 1.1352 \\
7.0152 & 1.1361 & 7.0166 & 1.1359 & - & - & - & - \\
\hline 0.6116 & 2.5745 & 0.6106 & 2.5789 & 0.6115 & 2.5745 & 0.6106 & 2.5790 \\
1.8318 & 2.5745 & 1.8313 & 2.5753 & 1.8318 & 2.5745 & 1.8313 & 2.5753 \\
3.0521 & 2.5745 & 3.0519 & 2.5747 & - & - & - & - \\
\hline
\end{tabular}

Table 2: A selection of antisymmetric trapped modes. For all calculations, $\ell s=0$, $s / a_{1}=s / a_{2}=1.2$. For the finite depth calculations $a_{1} / h=0.1$.

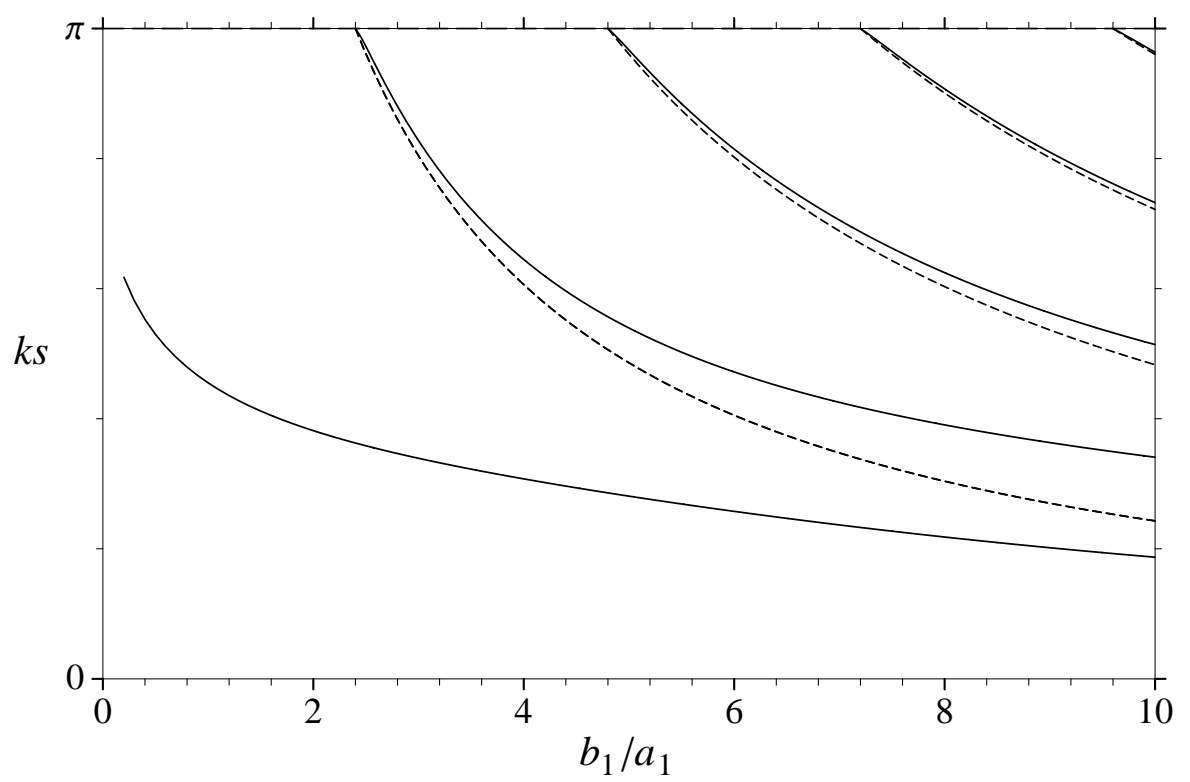

Figure 5: Lines in $k s-b_{1} / a_{2}$ parameter space where velocity conditions are satisfied; equation (123), - - - equation (124). Here $\ell h=0, a_{1} / h=a_{2} / h=0.1$, $s / h=0.24$.

are further zeros of transmission when $2 \pi<k s<3 \pi$ and so there are very likely further trapped modes within this range of $k s$. However, it becomes increasingly difficult to compute reliable results for $k s>2 \pi$ (for the parameters used here at least).

Uniqueness of the four barrier problem was established in section 5 for ranges of frequency determined by the inequalities (83) which are illustrated in figure 6 . Regions for which uniqueness of 


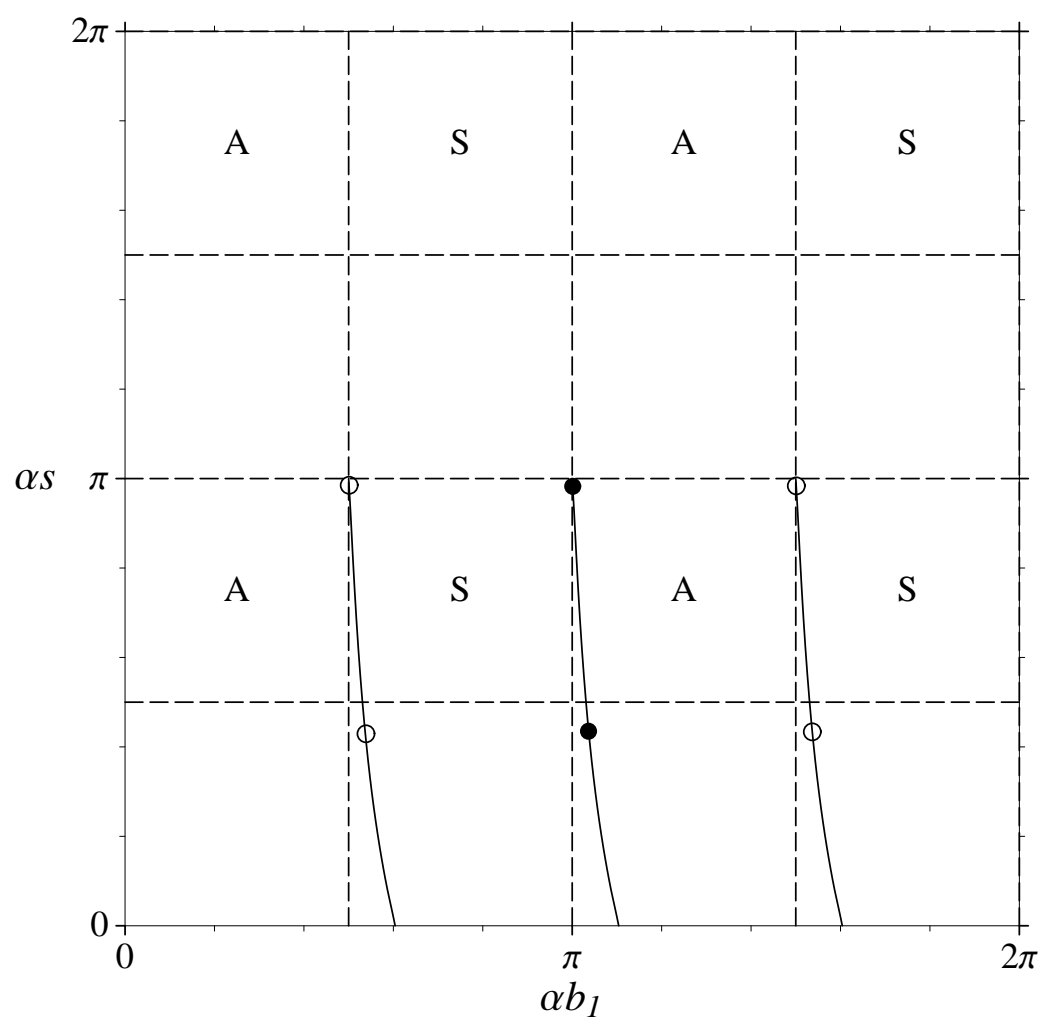

Figure 6: Regions of uniqueness for symmetric modes (S) and antisymmetric modes (A) determined by the inequalities (83). Computed trapped mode solutions are marked $\bullet$ for symmetric modes and $\circ$ for antisymmetric modes. The solid curves mark the predicted location of trapped modes based on the wide-spacing approximation.

symmetric modes has been established are denoted by $\mathrm{S}$ and regions for which uniqueness of antisymmetric modes has been established are denoted by A (the boundaries of each cell are included). Also included are some trapped mode solutions taken from tables 1 and 2. The locations of these are entirely consistent with the uniqueness theorem; modes that appear to lie on a line of constant $\alpha b_{1}$ are in fact slightly to the right and hence in agreement with the theorem. Also plotted in figure 6 are lines where trapped modes are approximately expected to occur in the two-dimensional problem $(\ell=0)$ based on the wide-spacing approximation for infinite-depth water. The limiting values for $K s=0$ are calculated from the asymptotic solution for two barriers found by Newman (1974). Although not shown in figure 6, the wide-spacing approximation predicts the existence of trapped modes for $0<\alpha b_{1}<\pi / 2$, but no modes have been located within this range using either numerical method. Perhaps this is not surprising as the wide-spacing approximation can be expected to break down for very small spacings between the barrier pairs. 


\begin{tabular}{|c|c|c|cc|}
\hline$a_{1} / h$ & $a_{2} / h$ & $s / h$ & $b_{1} / a_{1}$ & $K a_{1}$ \\
\hline 0.10 & 0.10 & 0.12 & 2.8592 & 1.1388 \\
0.10 & 0.12 & 0.12 & 3.2992 & 1.0046 \\
0.12 & 0.10 & 0.12 & 3.2234 & 1.2055 \\
0.10 & 0.10 & 0.10 & 3.0857 & 1.0656 \\
\hline
\end{tabular}

Table 3: Effect on a symmetric trapped mode of changes in the geometry: $\ell h=0$.

\begin{tabular}{|c|cc|cc|cc|cc|}
\hline$\ell h$ & $b_{1} / a_{1}$ & $K a_{1}$ & $b_{1} / a_{1}$ & $K a_{1}$ & $b_{1} / a_{1}$ & $K a_{1}$ & $b_{1} / a_{1}$ & $K a_{1}$ \\
\hline 0 & 2.8592 & 1.1388 & 5.6304 & 1.1365 & 1.2226 & 2.5726 & 2.4423 & 2.5742 \\
1 & 2.8615 & 1.1422 & 5.6349 & 1.1399 & 1.2226 & 2.5745 & 2.4423 & 2.5760 \\
2 & 2.8676 & 1.1525 & 5.6465 & 1.1505 & 1.2227 & 2.5801 & 2.4424 & 2.5818 \\
3 & 2.8760 & 1.1701 & 5.6626 & 1.1685 & 1.2227 & 2.5898 & 2.4425 & 2.5913 \\
4 & 2.8856 & 1.1951 & 5.6819 & 1.1939 & 1.2227 & 2.6032 & 2.4428 & 2.6045 \\
5 & 2.8961 & 1.2272 & 5.7039 & 1.2264 & 1.2229 & 2.6200 & 2.4431 & 2.6214 \\
\hline
\end{tabular}

Table 4: Effect on selected symmetric modes of changes in $\ell h: s / a_{1}=s / a_{2}=1.2, a_{1} / h=0.1$.

From the work of Porter \& Evans (1995) and McIver (1985) it is known that zeros of transmission for a pair of vertical barriers will persist if the geometry is perturbed. Thus, for a configuration where there is known to be a zero of transmission at a certain frequency, if the length of one or both barriers, or the spacing between the barriers, is changed then in general there will still be zero of transmission at a nearby frequency. The only exception is that the zero may be lost if the spacing is increased by too much (see Porter \& Evans 1995, figure 8). This suggest that the existence of trapped modes for two barrier pairs may be preserved by similar perturbations. This is indeed the case and a small number of calculations are given in table 3 to illustrate this point.

All of the calculations reported so far have been for the two-dimensional case, $\ell=0$. Exactly the same procedures may be applied to find trapped modes for $\ell \neq 0$ and a few results are given in table 4 to show the effect of increasing $\ell$ on some of the symmetric modes already given in table 1 . The general trend is that both the spacing $b_{1} / a_{1}$ and the frequency parameter $K a_{1}$ increase as $\ell h$ is increased. The changes in $b_{1} / a_{1}$ are quite small for modes with higher frequencies. 


\section{Discussion}

The geometry in figure 1 provides the first example of two surface-piercing structures separated by a bounded portion of the free surface for which the uniqueness theorem holds for all $K>\ell \tanh \ell h$, that is for the the whole continuous spectrum. Previously, the uniqueness of the solution in this situation has been established only for some frequency intervals. For the two-dimensional problem $(\ell=0)$, the first uniqueness theorem was proved by Kuznetsov (1988) and extended by Kuznetsov \& Simon (1995); however their frequency interval (which depends on the geometry) for uniqueness is finite. For the oblique case $(\ell>0)$ with two symmetric surface-piercing structures, Kuznetsov at al. (1998) generalised a method previously used in two dimensions (see the appendix to Linton \& Kuznetsov 1997) to prove uniqueness of symmetric and antisymmetric solutions when $\alpha b$ belongs to complementary intervals ( $2 b$ is the distance between structures satisfying John's condition). For $0<K<\ell \tanh \ell h$, the uniqueness proof of McIver (1991) and Simon (1992) for a single surfacepiercing cylinder has been noted to hold (Kuznetsov et al. 1998) for an arbitrary finite number of cylinders each satisfying John's condition.

For the two-dimensional problem $(\ell=0)$, there are only two criteria of uniqueness for totally submerged structures known at the present time. That of Maz'ya (1978), subsequently extended by Kuznetsov (1991), does not apply for any geometry with two submerged barriers. The uniqueness theorem of Simon \& Ursell (1984) holds for pairs of submerged barriers that are not too widely spaced or do not have top points too close to the free surface; hence not all possible cases of two-barrier geometries are covered by this theorem.

Thus, the present uniqueness theorem for two vertical barriers extends in two ways previous work on two surface-piercing structures. First of all it provides a new example for the two-dimensional water-wave problem $(\ell=0)$ in which uniqueness has been established for the whole continuous spectrum. Secondly the two-barrier geometry is the first for which uniqueness has been proven for the whole continuous spectrum for any $\ell>0$.

Also, uniqueness is established for all $K>0$ in the two-dimensional problem $(\ell=0)$ of three symmetrically-arranged surface-piercing barriers. This is the first of example of a uniqueness theorem that holds for all frequencies in the presence of three bodies floating in the free surface. For $\ell>0$ and an arbitrary arrangement of three barriers an infinite sequence of uniqueness intervals is found.

In the case of four symmetrically arranged barriers, strong numerical evidence has been pro- 
vided for the existence of trapped modes in frequency intervals complementary to those for which uniqueness is proved. The evidence is equally strong that these trapped modes are associated with zeros of transmission for a pair of barriers. A natural topic for further research is to investigate the construction of trapped modes for other geometries for which zeros of transmission are known to exist.

Given a pair of barriers for which a zero of transmission exists, trapped modes for two such pairs are found only for certain discrete spacings between the pairs. For any small perturbation of the spacing the trapped mode no longer exists. However, it is interesting that for any other perturbation of the geometry (changing the length of one or both barriers in, or the spacing of, the pair) it is possible to find a trapped mode at a frequency near to that for the unperturbed geometry.

\section{Acknowledgment}

This work was carried out during a visit to Loughborough University by Dr. Kuznetsov. The financial support of the London Mathematical Society and Loughborough University is gratefully acknowledged.

\section{References}

Evans, D. V. \& Morris, C. A. N. 1972 Complementary approximations to the solution of a problem in water waves. J. Inst. Maths Applic. 10, 1-9.

Evans, D. V. \& Porter, R. 1998 A example of non-uniqueness in the two-dimensional linear waterwave problem involving a submerged body. Proc. Roy. Soc. Lond. A 454, 3145-3165.

Jarvis, R. J. 1971 The scattering of surface waves by two vertical plane barriers. J. Inst. Maths Applic., 7, 207-215.

John, F. 1950 On the motion of floating bodies, II. Commun. Pure Appl. Maths 3, 45-101.

Kozlov, V. A., Maz'ya, V. G. \& Rossman, J. 1997 Elliptic boundary value problems in domains with point singularities. AMS, Providence.

Kuznetsov, N. G. 1988 Plane problem of the steady-state oscillations of a fluid in the presence of two semiimmersed cylinders. Math. Notes Acad. Sci. USSR 44, 685-690.

Kuznetsov, N. G. 1991 Uniqueness of a solution of a linear problem for stationary oscillations of a liquid. Diffl. Equat. 27, 187-194. (Translated from Russian.) 
Kuznetsov, N., Porter, R., Evans, D. V. \& Simon, M. J. 1998 Uniqueness and trapped modes for surface-piercing cylinders in oblique waves. J. Fluid Mech. 365, 351-368.

Kuznetsov, N. \& Porter, R. 1999 Uniqueness and trapped modes in the two-dimensional waterwave problem. Euro. J. Appl. Maths (submitted).

Kuznetsov, N. G. \& Simon, M. J. 1995a On uniqueness in linearized two-dimensional water-wave problem for two surface-piercing bodies. Q. J. Mech. Appl. Math. 48, 507-515.

Kuznetsov, N. G. \& Simon, M. J. 1995b On uniqueness in the two-dimensional water-wave problem for surface-piercing bodies in fluid of finite depth. Appl. Math. Report No. 95/4, Dept. of Math., University of Manchester.

Levine, H. \& Rodemich, E. 1958 Scattering of surface waves in an ideal fluid. Math. and Stat. Lab. Tech. Report No. 78, Stanford University.

Linton, C. M. \& Kuznetsov, N. G. 1997 Non-uniqueness in two-dimensional water wave problems: numerical evidence and geometrical restrictions. Proc. Roy. Soc. Lond. A 453, 2437-2460.

Martin, P. A. 1991 End-point behaviour of solutions to hypersingular integral equations. Proc. Roy. Soc. Lond. A 432, 301-320.

Maz'ya, V. G. 1978 Solvability of the problem on the oscillations of a fluid containing a submerged body. J. Soviet Maths 10, 86-89.

McIver, M. 1996 An example of non-uniqueness in the two-dimensional linear water-wave problem. J. Fluid Mech. 315, 257-266.

McIver, M. 1997 . Resonance in the unbounded water wave problem. In Proceedings of the 'Twelfth International Workshop on Water Waves and Floating Bodies' held in Carry-le-Rouet, France, 16-19 March 1997, 183-187.

McIver, P. 1985 Scattering of surface waves by two surface-piercing vertical barriers. IMA J. Appl. Math. 35, 1-17.

McIver, P. 1991 Trapping of surface water waves by fixed bodies in channels. Q. J. Mech. Appl. Math. 44, 193-208.

Newman, J. N. 1974 Interaction of water waves with two closely spaced vertical barriers. J. Fluid Mech. 66, 97-106.

Parsons, N. F. \& Martin, P. M. 1992 Scattering of water waves by submerged plates using hypersingular integral equations. Appl. Ocean Res. 14, 313-321.

Parsons, N. F. \& Martin, P. M. 1994 Scattering of water waves by submerged curved plates and by surface-piercing flat plates. Appl. Ocean Res. 16, 129-139. 
Parsons, N. F. \& Martin, P. M. 1995 Trapping of water waves by submerged plates using hypersingular integral equations. J. Fluid Mech. 284, 359-375.

Porter, R. \& Evans, D. V. 1995 Complementary approximations to wave scattering by vertical barriers. J. Fluid Mech. 294, 155-180.

Simon, M. J. \& Ursell, F. 1984 Uniqueness in linearised two-dimensional water-wave problems. J. Fluid Mech. 148, 137-154.

Simon, M. J. 1992 On a bound for the frequency of surface waves trapped near a cylinder spanning a channel. Theoret. Comput. Fluid Dynamics. 4, 71-78.

Wigley, N. G. 1964 Asymptotic expansions at a corner of solutions of mixed boundary value problems. J. Math. Mech. 11, 549-576.

Yu, Y. S. \& Ursell, F. 1961 Surface waves generated by an oscillating circular cylinder on water of finite depth: theory and experiment. J. Fluid Mech. 11, 529-551. 